Check for updates

Cite this: RSC Adv., 2019, 9, 13838

Received 29th January 2019 Accepted 24th April 2019

DOI: $10.1039 / c 9 r a 00699 k$

rsc.li/rsc-advances

\section{Fundamentals and applications of acrylamide based microgels and their hybrids: a review}

\author{
Robina Begum, ${ }^{\text {ab }}$ Zahoor H. Farooqi, (iD *a Ejaz Ahmed, ${ }^{\text {a }}$ Ahsan Sharif, ${ }^{\text {a Weitai Wu (D) }}{ }^{\mathrm{c}}$ \\ and Ahmad Irfan ${ }^{\text {de }}$
}

Acrylamide based microgels have gained a lot of attention in the last three decades due to their potential applications in various fields based on their responsive behavior and chemical stability. In this article, the synthesis, properties, and applications of poly( $\mathrm{N}$-isopropylacrylamide-co-acrylamide) [P(NIPAM-Am)] microgels and P(NIPAM-Am) microgels having an additional ionic moiety in their network [P(NIPAM-Am(M)] are reviewed. These microgels may swell/deswell reversibly with slight changes in environmental conditions such as change in temperature/pH/ionic strength etc. of the medium. This responsive behavior makes the microgels a potential candidate for use in the field of nanotechnology, drug delivery, sensing and catalysis. A critical overview of the recent research progress in this area along with future perspectives is presented. The discussion is concluded with suggested possible future studies for further development in this area.

\section{Introduction}

IInstitute of Chemistry, University of the Punjab, New Campus, Lahore 54590, Pakistan.E-mail: zhfarooqi@gmail.com; Zahoor.chem@pu.edu.pk

${ }^{b}$ Centre for Undergraduate Studies, University of the Punjab, Lahore 54590, Pakistan ${ }^{\prime}$ State Key Laboratory for Physical Chemistry of Solid Surfaces, Collaborative Innovation Center of Chemistry for Energy Materials, The Key Laboratory for Chemical Biology of Fujian Province, Department of Chemistry, College of Chemistry and Chemical Engineering, Xiamen University, Xiamen 361005, China

${ }^{d}$ Research Center for Advance Materials Science, King Khalid University, P. O. Box 9004, Abha 61413, Saudi Arabia

${ }^{e}$ Department of Chemistry, Faculty of Science, King Khalid University, P. O. Box 9004, Abha 61413, Saudi Arabia
Robina Begum is working as a lecturer in Chemistry at the Centre for Undergraduate Studies, University of the Punjab since 2013. She is doing PhD in Chemistry under the supervision of Dr Ejaz Ahmed and Dr Ahsan Sharif at the Institute of Chemistry, University of the Punjab, Lahore. She carried out a part of her research work in the laboratory of Prof. Jianliang Xiao at the Department of Chemistry, University of Liverpool, UK as a Split-Site PhD scholar funded by the Commonwealth Scholarship Commission, UK 2017. Her research area is organic-inorganic hybrid materials for various applications.

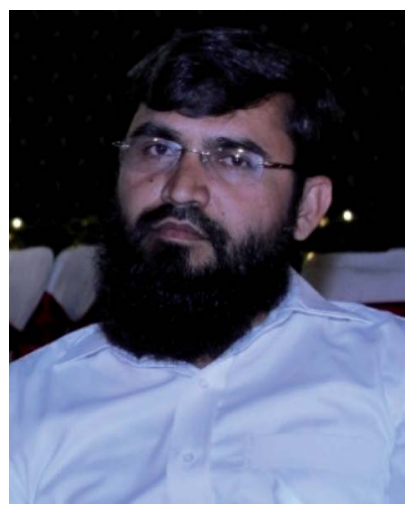

Dr Zahoor Hussain Farooqi is currently working as an Assistant Professor of Physical Chemistry at the Institute of Chemistry, University of the Punjab, Lahore, Pakistan. He worked as a research associate at CSI, The City University of New York, USA under the Pak-US Science and Technology Cooperative Program from January 2009 to March 2010. He worked as a Honorary Research Fellow in the Department of Chemistry, University of Liverpool, UK from 01-05-2018 to 06-10-2018. His area of research is polymer colloid loaded nanoparticles for environmental and catalytic applications. 
have been widely reported in literature..$^{2-6}$ These microgels are temperature responsive in nature and show rapid swelling/ deswelling near a certain temperature which is called the volume phase transition temperature (VPTT). ${ }^{1}$ Poly $(N$-isopropylacrylamide) $[\mathrm{P}(\mathrm{NIPAM})]$ is the most common temperature responsive polymer microgel system and its VPTT in aqueous medium is $32{ }^{\circ} \mathrm{C}^{7}$ When NIPAM is copolymerized with Am, the hydrophilicity of the resultant polymeric network is increased and VPTT of the microgel system is shifted to higher temperature because Am units have more affinity towards water molecules as compared to NIPAM and it is difficult to remove water content from polymeric network made of both NIPAM and Am as compared to that made of NIPAM only. ${ }^{8}$ Presently, these microgels are being largely used in bio-sensing, ,,10 drug delivery, $^{11,12}$ catalysis $^{13}$ and optical transduction. ${ }^{14,15}$ These microgels are the most promising responsive polymers that remain intact in both swollen and de-swollen states. The size of microgel particles is smaller in deswollen state as compared to

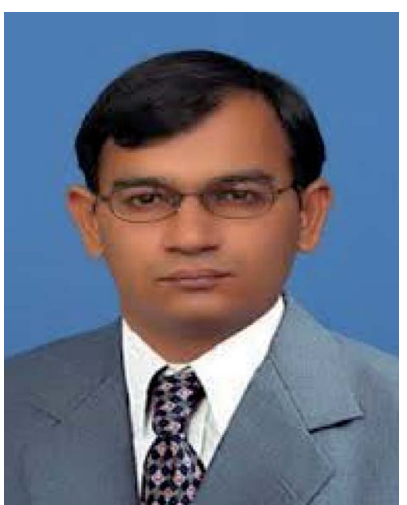

Dr Ejaz Ahmed is an Assistant Professor at the Institute of Chemistry, University of the Punjab, Lahore, Pakistan. He obtained his M.Sc degree in Chemistry from The Islamia University Bahawalpur and his PhD degree in Organic Chemistry from the University of Karachi, Karachi, Pakistan. He is a former manager of publications at Bentham Science publishers ltd and Journal of Chemical Society of Pakistan. His research interests include the isolation and structural elucidation of bioactive natural products from Marine and terrestrial medical plants. He is also interested in polymer hydrogels for environmental and catalytic applications.

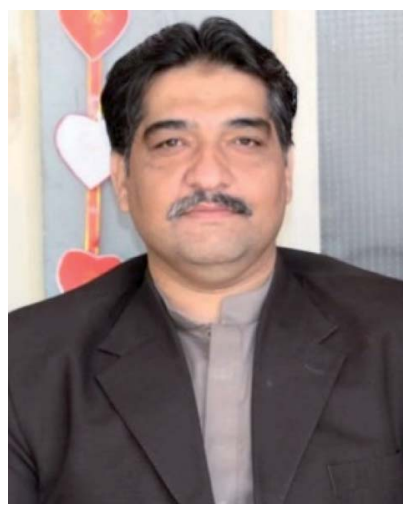

Dr Ahsan Sharif is an Assistant Professor at the Institute of Chemistry, University of the Punjab, Lahore, Pakistan. He obtained his M.Sc degree in Chemistry from Quaid-I-Azam University Islamabad, Pakistan in 2001. He obtained PhD in Organic Chemistry from HEJ Research Institute of Chemistry, Karachi, Pakistan. During his PhD, he spent two years at Cambridge University, UK where he did research work in the area of Supramolecular Chemistry. His research interests include the isolation and structural elucidation of bioactive natural products from Marine and terrestrial medicinal plants. He is also interested in polymer hydrogels for environmental and catalytic applications. that of swollen state. ${ }^{\mathbf{1 6}}$ These microgels possess large volume for transportation/storage of various molecules. The size of mesh sieves of these microgels are in nano range, so these polymer materials can be used as templates for synthesis of metal nanoparticles. ${ }^{17,18}$ Metal nanoparticles can also be successfully stabilized within microgels due to donor-acceptor interaction of functionalities of polymeric system and metal nanoparticles. Size of metal nanoparticles can be controlled by controlling crosslinking density of such kind of microgel particles. NIPAM is thermo-responsive in nature which is co-polymerized with Am using $N, N$-methylenebisacrylamide (MBAAm) as cross linker to increase water holding capacity of the microgels. ${ }^{4,19-21} \mathrm{Am}$ is a hydrophilic co-monomer ${ }^{5}$ and generally used in small quantity as compared to main monomer (mostly NIPAM), so it does not alter the original properties of NIPAM based polymer microgels significantly in case of its low mol percentage in feed composition of microgels but high content of Am may affect the responsive behavior of resulting microgels. ${ }^{13}$ Moreover,

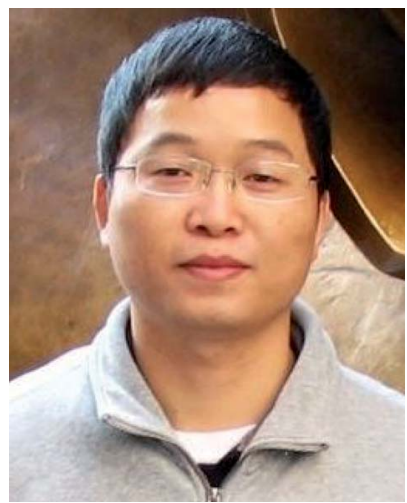

Weitai $W u$ received $B S$ (2003) and $\mathrm{PhD}$ (2008) degrees from the University of Science and Technology of China. In 2006 and 2007, he worked as a research staff member at the Hong Kong Polytechnic University. He then worked as a postdoctoral research associate at the City University of New York until 2011. He is currently a full professor of chemistry at Xiamen University, China. His research group is currently working on the chemistry and physics of smart materials, and their applications in the field of biomedicine, energy, and environment.

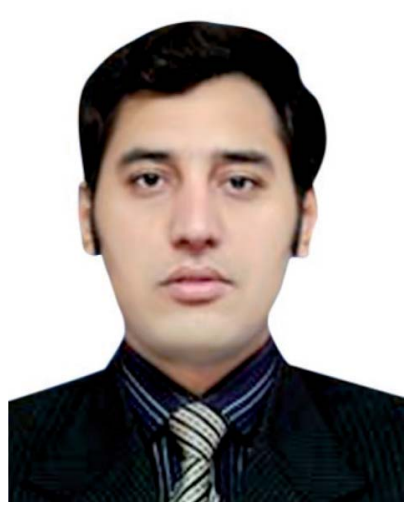

Dr Irfan Ahmad graduated from GCES, University of the Punjab (PU) in 2002 and received his M.Sc. (2004) from UAF. Through a mutual scholarship from MOE, Pakistan and CSC, China, he received his $P h D$ (2010) from NENU, China. He worked as an Assistant Professor at the University of the Punjab, Lahore Pakistan and King Khalid University (KKU), Saudi Arabia. He is currently serving as an Associate Professor in the Department of Chemistry and RCAMS, KKU. His present research interests are advanced functional materials, nanotechnology, catalysis, renewable energy, semiconductors and drug designing. 
addition of Am does not increase the charge density onto the copolymer microgels to large extent due to which $\operatorname{poly}(\mathrm{N}$ isopropylacrylamide-acrylamide $) \quad[\mathrm{P}(\mathrm{NIPAM}-\mathrm{Am})]$ microgels remain non-responsive towards the $\mathrm{pH}$ of the medium except in very low $\mathrm{pH}$ values ${ }^{2}$ due to which these microgels do not lose their temperature sensitivity in a wide $\mathrm{pH}$ range. NIPAM and Am can also be polymerized with other ionic co-monomers like vinylacetic acid (VAA), ${ }^{22}$ phenyl boronic acid (PBA), ${ }^{23}$ acrylic acid (AA), ${ }^{5}$ methacrylic acid (MAA) ${ }^{24}$ to diversify their properties depending upon their application. In this review, we will mainly focus on P(NIPAM-Am) microgels. However some examples of NIPAM and Am based microgels having some additional ionic moiety in their network have been also included in this survey. The acrylamide functional group in the polymeric network is the least reactive due to which P(NIPAM-Am) microgels can be treated as inert microgel systems. The inertness of these microgel systems make them a potential candidate for the carrier of inorganic nanoparticles to be used as catalysts in various organic reactions. The addition of Am gives thermal stability to P(NIPAM-Am) microgels in aqueous medium due to its hydrophilic nature. P(NIPAM-Am) microgels have a potential to be used in catalysis due to their thermo-responsive behavior, inertness, thermal stability, open network, ligand characteristics of their functionalities, high water holding capacity, nonionic nature and hydrophilicity in a wide temperature range and have gained a lot of interest of researchers in the last two decades. ${ }^{\mathbf{1 4 2 3 , 2 5 , 2 6}}$ The polymerization of NIPAM and Am with an ionic monomer does not only increase the hydrophilicity in the network but also gives multi-responsiveness to the polymeric network. ${ }^{27}$ Present progress of P(NIPAM-Am)/P(NIPAM-Am-IM) microgel systems has not been reviewed critically in literature. To the best of our knowledge, properties and applications of $\mathrm{P}($ NIPAM-Am)/P(NIPAM-Am-IM) microgels have not been reported in a systematic way in the form of a review article previously. Although many reviews on smart polymer microgels published by $\mathrm{us}^{28}$ and others ${ }^{29}$ are available in literature but all of those are general reviews and do not deal with this particular system of interest with respect to their properties and applications. For example Saunders et al. reviewed properties and biomedical applications of a wide variety of responsive microgels including P(NIPAM) microgels. ${ }^{30}$ It is an excellent review on use of microgel based biomaterials but it was written ten years back and is out of date now. Moreover it is a general review and covers all types of microgels. Progress in synthesis, characterization and applications of poly $\left(\mathrm{N}\right.$-isopropylacrylamide- $\mathrm{Co}^{-}$ acrylic acid) [P(NIPAM-AA)] microgels has been reviewed by our group recently ${ }^{28}$ but this review is related to microgels composed of NIPAM and AA monomers. Our group has been also presented development of core-shell microgels with polystyrene core and $\mathrm{N}$-isopropylacrylamide based shell in the form of a review ${ }^{31}$ but microgel systems made of NIPAM and Am are not included in this report. Naseem et al. has published a review ${ }^{32}$ which emphasized on vinyl acetic acid based microgels only. Similarly a comprehensive review on responsive behavior of poly(vinylcaprolactam) has been published by Cortez-Lemus and coworkers ${ }^{33}$ which covers $N$-vinylcaprolactam based microgels only. Most updated, comprehensive and informative report on functional polymer microgels including few examples of acrylamide system in the form of an account has been published by Plamper et al. ${ }^{34}$ but it is also a general over view of all types of polymer microgels.

Here in, recent developments in methodologies adopted to synthesize P(NIPAM-Am) based mono-disperse microgel particles, their properties and applications have been reported. Section 1 gives a brief introduction of the topic. In Section 2, methodologies adopted to synthesize P(NIPAM-Am)/P(NIPAMAm-IM) microgels have been discussed. In Section 3, different characterization techniques used to analyze P(NIPAM-Am)/ P(NIPAM-Am-IM) microgels have been described briefly. In Section 4, temperature responsive, phase transition behavior as well as effect of acrylamide, crosslinker and surfactant feed contents on properties of these specific microgels have been discussed in detail. Applications of P(NIPAM-Am) microgels in fabrication and stabilization of inorganic nanoparticles, photonics, biomedical field and catalysis has been discussed in detail in Section 5. Summary and future aspects of this specific polymer microgel system have also been enlightened at the end of this review article in Section 6.

\section{Synthesis of P(NIPAM-Am)/ P(NIPAM-Am-IM) polymer microgels}

\subsection{Free radical precipitation polymerization}

The most common method used for synthesis of P(NIPAM-Am)/ $\mathrm{P}$ (NIPAm-Am-IM) microgels is free radical precipitation polymerization. ${ }^{13}$ Potassium per sulfate (KPS), ${ }^{12}$ ammonium per sulfate (APS) ${ }^{13}$ or azodiisobutyronitrile $(\mathrm{AiBN})^{8}$ have been reported as initiators in this method of polymerization using sodium dodecyl sulfate (SDS) as emulsifier. In this methodology, NIPAM (monomer), Am (comonomer), additional ionic monomer (if any), MBAAm (crosslinker) and SDS (surfactant) are dissolved in water. Reaction mixture is stirred to dissolve reactants in water and purged with nitrogen to remove dissolved oxygen at room temperature. Then, temperature is raised up to $70{ }^{\circ} \mathrm{C}$ and reaction mixture is further stirred at $70{ }^{\circ} \mathrm{C}$ for half an hour under continuous condensing with nitrogen supply. Then initiator generally KPS or APS is added to initiate the polymerization process. The reaction mixture turns turbid after few minutes of addition of initiator which is a sign of the onset of polymerization. Reaction is continued further for 4-6 hours under nitrogen gas purging and stirring at $70{ }^{\circ} \mathrm{C} .{ }^{12}$ The dispersion of microgel particles is centrifuged and re-dispersed again in de-ionized water to obtain mono-disperse microgel particles. To remove surfactant, initiator and unreacted monomers, synthesized P(NIPAM-Am)/P(NIPAM-Am-IM) microgels are dialyzed against distilled water. The general scheme for synthesis of P(NIPAM-Am) microgels by precipitation polymerization is in shown in Fig. 1. Majority of the scientists have reported this method of synthesis of uniformly crosslinked $\mathrm{P}($ NIPAM-Am) microgel particles with slight variation in methodology. ${ }^{4,35,36}$ Hoare et al. synthesized P(NIPAM-Am) microgels by free radical precipitation polymerization as described above and reported that this is the best method to obtain mono- 

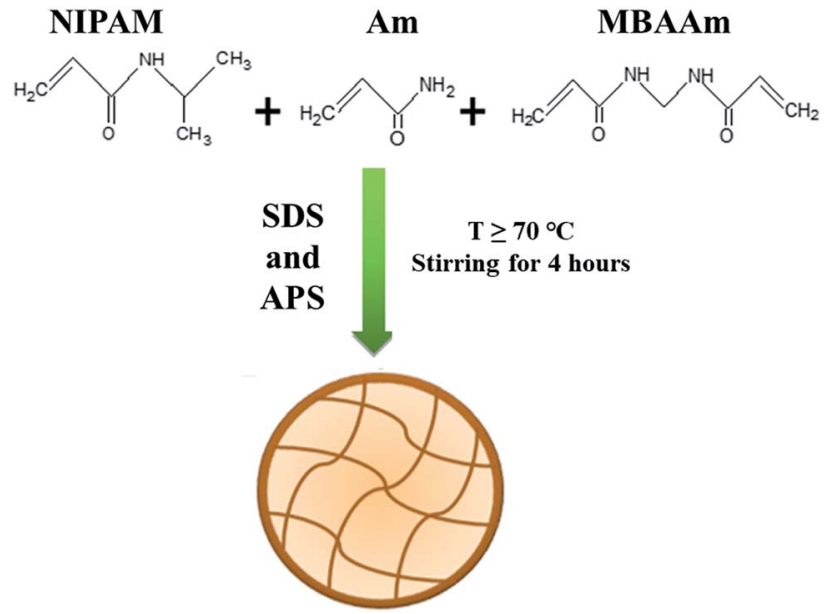

Fig. 1 Schematic representation of synthesis of poly $(N$-isopropylacrylamide-acrylamide) [P(NiPAM-Am)] microgel by precipitation polymerization method.

disperse microgel particles. ${ }^{2}$ NIPAM, Am and crosslinker were successfully incorporated into the polymer microgels in stoichiometric amounts corresponding to their feed ratios. They also confirmed that NIPAM and Am were incorporated uniformly throughout P(NIPAM-Am) polymer network. It means that $\mathrm{P}(\mathrm{NIPAM}-\mathrm{Am})$ particles of uniform composition can be obtained by precipitation polymerization of NIPAM and Am monomers using MBAAm as cross linker. They also prepared P(NIPAM-Am-MAA) microgels using the same methodology but distribution of methacrylic acid units (ionic co-monomer) was not found to be uniform throughout the polymer network by precipitation polymerization. ${ }^{2}$ This indicates that use of $\mathrm{P}$ (NIPAM-Am) microgel system is the best choice as compared to P(NIPAM-Am-MAA) microgel system where uniformity of monomer units in the network matters.

Zhang et al. synthesized a series of monodisperse P(NIPAMAm) microgels using different feed contents of Am in the presence of different amounts of SDS and reported that morphology and responsive behavior of the microgels can be easily tuned using free radical precipitation polymerization method of preparation of microgels. ${ }^{37}$ The similar observations have been also reported by Shen and coworkers. ${ }^{19}$ Synthesis of monodisperse $\mathrm{P}$ (NIPAM-Am-IM) microgel particles with different IM units like P(NIPAM-Am-AA), ${ }^{38} \mathrm{P}\left(\right.$ NIPAM-Am-MAA) ${ }^{39}$ and $\mathrm{P}(\mathrm{NIPAM}-\mathrm{Am}-\mathrm{VAA})^{17}$ by precipitation polymerization method described above has been reported in literature.

\subsection{Redox polymerization}

The free radical precipitation polymerization of NIPAM and Am is generally carried out at $70-80{ }^{\circ} \mathrm{C}$ in aqueous medium as described above but polymerization can be achieved successfully at low temperature by using some accelerator. The initiator and accelerator form a redox pair to initiate radical polymerization at low temperature and this method is known as redox polymerization. Synthesis of $\mathrm{P}$ (NIPAM-Am) hydrogels at $4{ }^{\circ} \mathrm{C}$ using sodium persulfate (NaPS) and $N, N, N^{\prime}, N^{\prime}-$ tetramethylethylene-diamine (TEMED) as redox pair in aqueous medium has been reported in literature. ${ }^{40}$

\subsection{Synthesis of microgel particles from P(NIPAM-Am) linear chain polymer}

Synthesis of biocompatible and biodegradable microgels having P(NIPAM-Am) polymer chain as a minor component have been also reported by Sung and coworkers using totally different methodology. ${ }^{11}$ In this method, P(NIPAM-Am) linear chain polymer was synthesized initially. Then polymer aqueous solution was mixed with gelatin sol phase solution and emulsified with silicone oil in the presence of a triblock copolymer surfactant [poly(ethylene glycol)-blockpoly(propylene glycol)-block-poly(ethylene glycol)]. Silicone oil was removed by repeated centrifugation of the mixture. After that, phosphate buffer solution containing a natural cross linker genipin was added into gelatin loaded with $\mathrm{P}(\mathrm{NIPAM}-\mathrm{Am})$. The gelatin based thermo-responsive microgels cross linked by genipin were used for controlled drug release. A similar method of synthesis of crosslinked microspheres from pre-formed linear thermo-responsive poly $(N$-isopropylacrylamide- $c o$-acrylamide- $c o$-hydroxyethyl acrylate) [P(NIPAM-co-Am-co-HEA)] system has been described by Fundueanu and coworkers. ${ }^{16}$ They determined composition of pre-formed $\mathrm{P}$ (NIPAM-co-Am-co-HEA) linear polymer using ${ }^{13} \mathrm{C}$ NMR spectroscopy. They used glutaraldehyde as crosslinker to convert linear polymers into microspheres.

\subsection{Radiation polymerization}

P(NIPAM-Am) hydrogels are also prepared by radiation polymerization at room temperature. ${ }^{41}$ Yong et al. prepared P(NIPAM-Am) copolymer hydrogels with different feed contents of NIPAM and Am in aqueous medium by using method of radiation polymerization. ${ }^{41}$ Co-60 gamma rays were used as source of irradiation. This method is used for synthesis of bulk hydrogels which grinded into small particles. Then these particles are dispersed in water for various applications. Moreover polymer particles made by this method are not monodisperse due to which their applications are limited.

\section{Characterization of P(NIPAM-Am) based polymer microgels}

Various techniques like Photo correlation spectroscopy, ${ }^{12}$ Transmission electron microscopy (TEM), ${ }^{8}$ Dynamic light scattering (DLS), ${ }_{1}^{1}$ UV-visible spectroscopy (UV-vis) ${ }^{13}$ Fourier transform infrared spectroscopy (FTIR), ${ }^{42}$ Scanning electron microscopy (SEM) ${ }^{43}$ Raman spectroscopy (RS), ${ }^{8}$ Energy dispersive X-ray spectroscopy (EDX) ${ }^{44}$ Nuclear magnetic resonance spectroscopy (NMR), ${ }^{45}$ Differential mechanical analysis (DMA), ${ }^{46}$ Laser light scattering spectrometry (LLS), ${ }^{9}$ Differential scanning calorimetry (DSC) ${ }^{13}$ and Atomic force microscopy (AFM) have been reported to characterize P(NIPAM-Am) microgel particles. Photon correlation spectroscopy/DLS is used for determination of hydrodynamic size and size 
distribution of polymer microgel particles. This technique is also used to investigate temperature and $\mathrm{pH}$ responsive behavior of P(NIPAM-Am) microgel system. Microscopic techniques like TEM, SEM and AFM are utilized for determination of morphologies of P(NIPAM-Am) microgel particles and their hybrids. ${ }^{47}$ NMR, FTIR and RS are widely used methods for identification of functionalities of the resulting polymer particles. ${ }^{48}$ EDX and XRD methods are applied to inorganic nanoparticles loaded acrylamide based microgels to confirm metallic nature of inorganic materials loaded in polymer microgels. ${ }^{49} \mathrm{UV}$ visible spectroscopy is used to determine VPTT of the P(NIPAMAm) microgels and their hybrids. Formation of Plasmonic nanoparticles inside the P(NIPAM-Am) microgel dispersion can be confirmed by UV visible spectroscopy. This technique is also useful for investigation of tuning of optical properties of P(NIPAM-Am) microgels loaded with Plasmonic nanoparticles. The progress of reaction catalyzed by metal nanoparticles loaded P(NIPAM-Am) microgels can be monitored by UV visible spectrophotometry. ${ }^{13}$ The potential of this technique for characterization of smart polymer microgels and their hybrids for different applications has been recently reviewed by our group. ${ }^{50}$ TGA, DSC and DMA are used for investigation of thermal stability of P(NIPAM-Am) microgels and their hybrids. TGA is also useful for determination of metal content in P(NIPAM-Am) microgels loaded with metal nanoparticles. ${ }^{13,51}$ The purpose of use of aforementioned characterization techniques has been described in detail in later sections.

\section{Properties of acrylamide based microgels}

\subsection{Temperature responsive behavior of acrylamide based microgels}

Microgel particles which show a sudden change in their size (measured in term of hydrodynamic radius or diameter) with the change in temperature of the medium are called thermoresponsive microgels and this behavior of microgels is called their thermo-sensitivity. P(NIPAM) microgels are well known temperature responsive microgels which shows sudden decrease in their size at $32{ }^{\circ} \mathrm{C}$ in aqueous medium and their thermo-responsive behavior has been widely reported in scientific literature. ${ }^{52-56} \mathrm{P}(\mathrm{NIPAM}-\mathrm{Am})$ microgels are also thermo-responsive due to presence of NIPAM content in their network. However Am is hydrophilic monomer and its polymerization with NIPAM may shift VPTT of resulting microgels to higher temperature in aqueous medium. Wang et al. measured hydrodynamic diameter of P(NIPAM-Am) microgels in the temperature range of $20-50{ }^{\circ} \mathrm{C}$ using DLS in aqueous medium and reported that the average hydrodynamic diameter of P(NIPAM-Am) microgels was decreased from 270 to $170 \mathrm{~nm}$ with the increase in temperature of the medium from 12 to $37{ }^{\circ} \mathrm{C}$ along with shift in VPTT depending upon feed content of microgels. ${ }^{1}$ Effect of feed content on thermo-sensitivity of $\mathrm{P}$ (NIPAM-Am) microgels has been discussed in later sections (4.3-4.6). They also investigated the size and shape variation of microgel particles with increase in temperature from 12 to $37^{\circ} \mathrm{C}$ using TEM analysis and found that morphology of microgel particles was changed from spherite to irregular shape along with decrease in their size as shown in Fig. 2. The aqueous dispersion of P(NIPAm-Am) microgels was placed on carbon coated copper grid and was allowed to dry for 24 hours at aforementioned values of temperature before TEM measurement. The irregularity in shape of microgel particles at elevated temperature may be due to heterogeneity in structure of polymer network while swelling of microgel particles at temperature $<$ VPTT is due to formation of hydrogen bonding between water and amide groups of polymer network. At temperature > LCST, hydrogen bonding between water and microgel contents was disrupted and hydrophobic interaction among the microgel contents was become stronger and as a result microgel particles were deswelled. The similar observations of variation in size of $\mathrm{P}$ (NIPAM-Am) microgel particles with change in temperature has been reported by other researchers. ${ }^{5,57,58}$ The polymerization of NIPAM, Am with an additional ionic monomer does not only increase the hydrophilicity in resulting microgels but also gives them multi-responsive behavior. Farooqi et al. investigated the variation in size of $\mathrm{P}$ (NIPAM-Am-AA) and P(NIPAM-Am-PBA) microgels with change in temperature, $\mathrm{pH}$ and ionic strength of medium and noted that both microgel systems shows variation in their size with aforementioned stimuli. ${ }^{10}$ They also observed that P(NIPAM-Am-AA) are thermo-responsive under low $\mathrm{pH}$ values and lose their temperature sensitivity at high $\mathrm{pH}$ values because of increase in hydrophilicity in polymer network as a result of ionization of carboxylic acid groups.

\section{2. $\mathrm{pH}$ responsive behavior of acrylamide based microgels}

The P(NIPAM-Am) microgel particles are considered irresponsive to change in a wide range of $\mathrm{pH}$ of the medium. For example $\mathrm{Wu}$ et al. measured hydrodynamic radius of P(NIPAMAm) microgel particles as a function of $\mathrm{pH}$ ranging from 2.5 to 8.5 in aqueous medium at $22{ }^{\circ} \mathrm{C}$ using dynamic light scattering measurement at scattering angle of $90{ }^{\circ} \mathrm{C}$ and found that value of $R_{\mathrm{h}}$ did not change with change in $\mathrm{pH}$ of the medium in aforementioned $\mathrm{pH}$ range. ${ }^{59}$ However under very low $\mathrm{pH}$ (in highly acidic medium), these microgel particles may get swell due to electrostatic repulsion among positively charged amide groups of polymeric network. But highly $\mathrm{pH}$ responsive Am based system can be obtained by copolymerization of NIPAM and $\mathrm{Am}$ with any ionic monomer. For example $\mathrm{Wu}$ and coworkers also studied $\mathrm{pH}$ responsive behavior of P(NIPAM-AmAA) microgels in the same $\mathrm{pH}$ range under similar conditions and found that the value of $R_{\mathrm{h}}$ increases with increase of $\mathrm{pH}$ of the medium and significant change was noted in the $\mathrm{pH}$ range of 3.5-4.5 ( $\mathrm{p} K_{\mathrm{a}}$ of AA is 4.2). ${ }^{59}$ Similarly Khan et al. reported $\mathrm{pH}$ responsive behavior of $\mathrm{P}(\mathrm{NIPAM}-\mathrm{Am}-\mathrm{VAA})$ microgels in aqueous medium. ${ }^{17}$ They found that the value of $R_{\mathrm{h}}$ of P(NIPAM-Am-VAA) microgel particles increases with increase in $\mathrm{pH}$ of the medium. The significant change in $R_{\mathrm{h}}$ of $\mathrm{P}$ (NIPAM-Am-VAA) microgel particles was observed in $\mathrm{pH}$ range of 4-5 which is very closed to $\mathrm{p} K_{\mathrm{a}}$ value of VAA ( $K_{\mathrm{a}}$ of VAA is 4.5). This increase in radius of particles may be attributed to electrostatic repulsion among charged moieties caused by protonation of carboxylic acid 

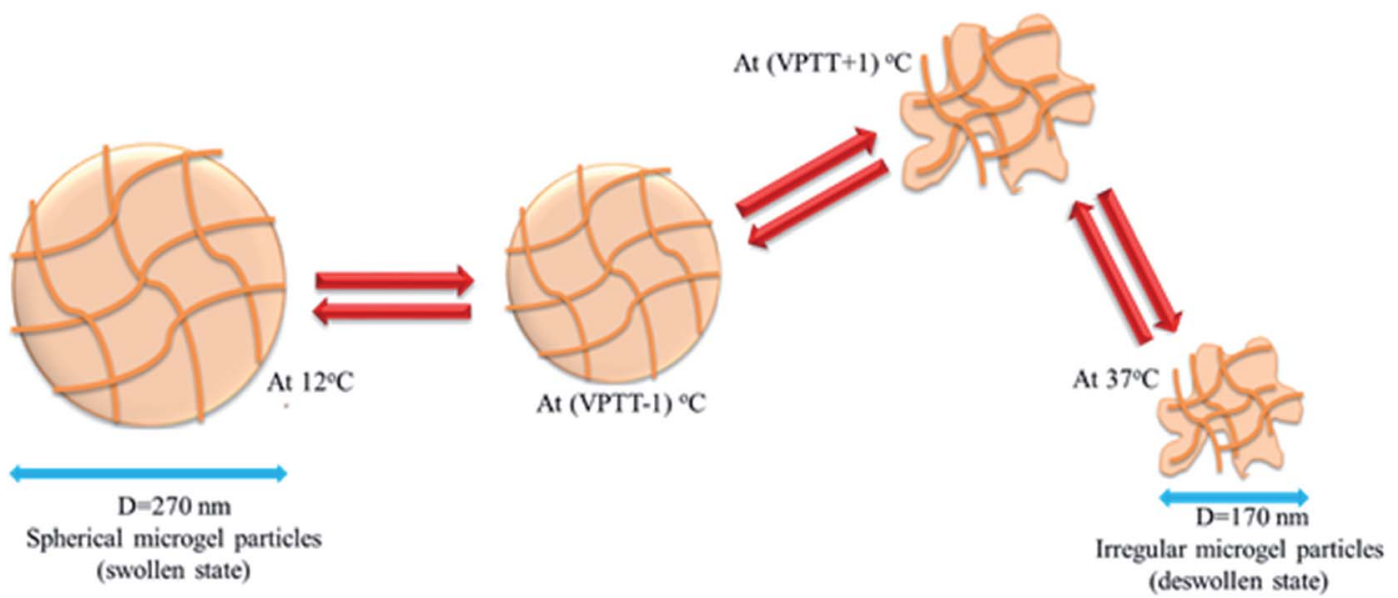

Fig. 2 The variation in size and shape of P(NIPAM-Am) microgel particles with change in temperature. ${ }^{1}$

groups of VAA units of polymeric network. They reported that the value of $R_{\mathrm{h}}$ becomes constant at $\mathrm{pH} \geq 5$ because no carboxylic acid is available for ionization and system becomes fully extended. The similar $\mathrm{pH}$ responsive trend of P(NIPAMAm-VAA), ${ }^{17} \quad$ P(NIPAM-Am-MAA) ${ }^{39}$ and $\quad$ P(NIPAM-Am-AA) ${ }^{59}$ microgels have been also reported in literature.

\subsection{Effect of acrylamide feed content on properties of P(NIPAM-Am) microgels}

The feed contents of P(NIPAM-Am) microgels have effect on responsive properties of resulting microgels. This effect is generally described in the form of deswelling ratio $(\alpha)$ which can be defined as

$$
\alpha=\left(\frac{V_{\mathrm{T}}}{V_{\text {swollen }}}\right)=\left(\frac{D_{\mathrm{T}}}{D_{20}}\right)^{3}
$$

where $V_{\mathrm{T}}$ and $V_{\text {swollen }}$ are the values of average volume of microgel particle at any temperature and in swollen state (at 20 ${ }^{\circ} \mathrm{C}$ ) respectively. $D_{\mathrm{T}}$ and $D_{20}$ are values of average hydrodynamic diameter at any temperature and at $20{ }^{\circ} \mathrm{C}$ (in swollen state) respectively. Values of equilibrium deswelling ratio of P(NIPAMAm) microgel samples Am-0, Am-10 and Am-15 with 0, 10 and $15 \mathrm{~mol}$ percent of Am at different temperatures of the medium reported by Wang et al. ${ }^{1}$ are listed in Table 1 . The time required

Table 1 Values of equilibrium deswelling ratio of P(NIPAM-Am) microgels with different mol percentages of NIPAM and Am at different values of temperature of the medium ${ }^{1}$

\begin{tabular}{llll}
\hline & \multicolumn{2}{l}{ Equilibrium deswelling ratio $(\alpha)$} \\
\cline { 2 - 4 } Temperature $\left({ }^{\circ} \mathrm{C}\right)$ & Am-0 & Am-10 & Am-15 \\
\hline 27.5 & 0.90 & 0.98 & 0.99 \\
30.0 & 0.83 & 0.91 & 0.93 \\
32.5 & 0.72 & 0.84 & 0.91 \\
37.2 & 0.39 & 0.45 & 0.75 \\
40.0 & 0.39 & 0.39 & 0.53
\end{tabular}

for swelling of P(NIPAM-Am) microgels is increased with increase in AAm feed content and time required for deswelling of P(NIPAM-Am) microgels is decreased with increase in AAm feed content (Fig. 3). When Am feed content of P(NIPAM-Am) microgels was increased, VPTT of the microgel system was shifted to high value of temperature. The value of LCST/VPTT of Am-0, Am-10 and Am-15 was found to be 32,34 and $38{ }^{\circ} \mathrm{C}$ respectively in aqueous medium. The increase in value of LCST with increase in Am content in P(NIPAM-Am) microgels has been also reported by Fang et al. ${ }^{60}$ who prepared P(NIPAM-Am) microgels with NIPAM : Am mol ratio of $83: 17$ for which value of LCST measured by UV-visible spectroscopy was found to be $40.1^{\circ} \mathrm{C}$. Budhlall et al. adjusted feed ratios of NIPAM and Am to obtain P(NIPAM-Am) microgels with VPTT temperature near to physiological temperature for designing of drug delivery system. ${ }^{4}$ Shen et al. also reported that P(NIPAM-Am) microgels with NIPAM : Am mol ratio of $9: 1$ has VPTT at $35{ }^{\circ} \mathrm{C}$ with a broaden transition range. ${ }^{19}$ Similar tuning of VPTT of $\mathrm{P}$ (NIPAM-Am) microgels has been also reported by Zhang et al. ${ }^{37}$ and Sanz et al. ${ }^{61}$ Thermo-sensitivity of microgel was decreased

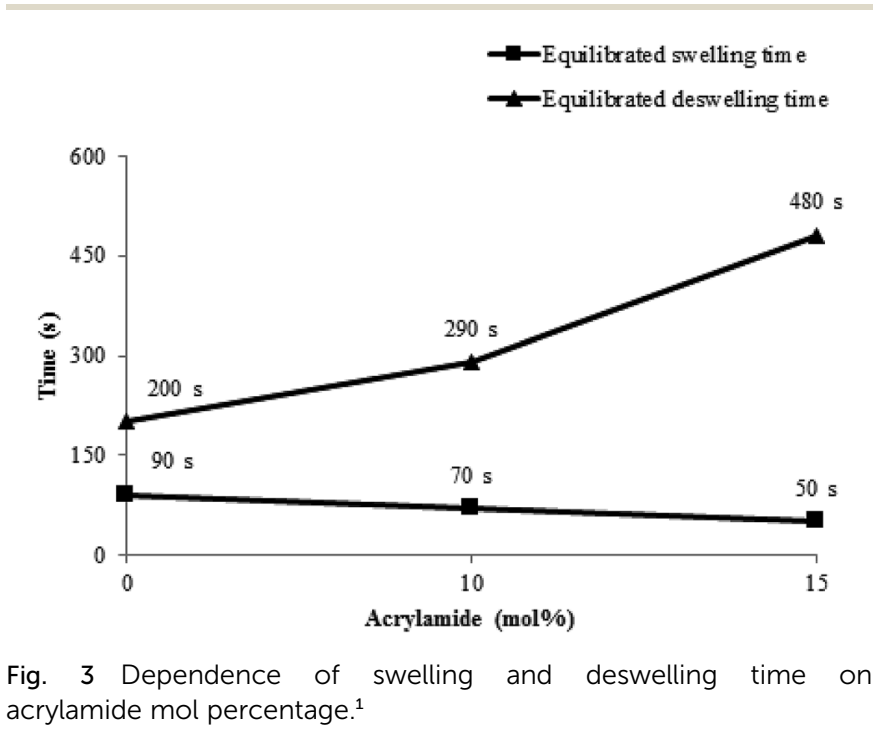


with increase of Am feed contents. The value of equilibrium deswelling ratio of $\mathrm{P}(\mathrm{NIPAM}-\mathrm{Am})$ polymer microgels measured by Wang et al. ${ }^{1}$ was decreased with increase of temperature of the medium as shown in Table 1.

\subsection{Effect of crosslinker feed content on properties of P(NIPAM-Am) based microgels}

Thermo-responsive properties of P(NIPAM-Am) microgels can also be tuned by changing the crosslinker feed content. Wang et al. also studied the effect of crosslinker feed content on thermo-responsive behavior of P(NIPAM-Am) microgel particles. ${ }^{62} \mathrm{P}$ (NIPAM-Am) microgels with 0.5 to $5.0 \mathrm{~mol}$ percent of crosslinker feed content showed abrupt change in hydrodynamic diameter in temperature range of $35-40{ }^{\circ} \mathrm{C}$ while microgels with 7.3 mol percent crosslinker do not show such type of behavior. Thus P(NIPAM-Am) microgels with low crosslinker feed content are flexible and more sensitive to temperature as compared to microgels with high crosslinker contents. It was also observed that size of microgel particles was decreased with increase of crosslinker feed contents.

\subsection{Effect of surfactant feed content on properties of P(NIPAM-Am) microgels}

The amount of surfactant used during synthesis also affects the properties of P(NIPAM-Am) microgels. Shen et al. ${ }^{19}$ fabricated P(NIPAM-Am) microgels with NIPAM : Am mol ratio of $9: 1$ in the presence of different amounts of SDS and noted that $R_{\mathrm{h}}$ decreases significantly with increase in feed concentration of SDS at constant feed contents of NIPAM and Am. They measured $R_{\mathrm{h}}$ of $\mathrm{P}$ (NIPAM-Am) microgels in aqueous medium at their VPTT $\left(37^{\circ} \mathrm{C}\right)$ using DLS as a function of mol percentage of SDS and noticed the decrease in $R_{\mathrm{h}}$ value with increase in concentration of SDS. Size regulation of P(NIPAM-Am) microgels by SDS has been also reported by various groups. ${ }^{37,63}$ Effect of nature and alkyl chain length of anionic surfactant on properties of $\mathrm{N}$-isopropylacrylamide, $\mathrm{N}$-isopropylmethacrylamide and $N$ - $n$-propylacrylamide based polymer microgels is beyond the scope of this review and can be seen in literature. ${ }^{64}$

\subsection{Effect of ionic strength on properties of P(NIPAM-Am) microgels}

It is very important to investigate the effect of ionic strength on properties of P(NIPAM-Am) microgels for their potential applications in biomedical field but unfortunately this aspect has not been described in literature in detail. Only a brief report on effect of ionic strength on the value of VPTT of P(NIPAM-Am) microgels is available in literature. ${ }^{37}$ Zhang and coworkers measured value of LCST/VPTT of P(NIPAM-Am) microgels in the presence of aqueous solution of different ionic strength using UV-visible spectroscopy and reported that value of LCST is decreased with increase in ionic strength due to well-known salting out effect. The value of LCST of P(NIPAM-Am) microgels with Am $15 \mathrm{wt} \%$ of the NIPAM was found to be decreased from $44{ }^{\circ} \mathrm{C}$ to $40{ }^{\circ} \mathrm{C}$ with increase of ionic strength from 0.0 to $0.16 \mathrm{M}$ in aqueous medium. ${ }^{37}$ It is needed to further explore the effect of ionic strength and nature of ions and solvent systems on responsive behavior and stability of P(NIPAM-Am) microgel particles in future studies.

\subsection{Phase transition behavior of P(NIPAM-Am) microgels}

$\mathrm{P}$ (NIPAM-Am) microgels show phase transition with change of temperature of the medium, content of acrylamide and crosslinker in the microgels. P(NIPAM-Am) microgel particles with 2.5 mol percent crosslinker showed four transition phases while microgel with 7.3 mol percent crosslinker showed three transition phases due to loss of flexibility and decrease of swelling ratio. Wang et al. observed these transitions of microgels phase induced by changing temperature. ${ }^{12}$ The microgel system was found to be a semi-translucent swollen gel at temperature below $22{ }^{\circ} \mathrm{C}$. Microgel was changed into clear, flowable suspension with change of temperature in range of $22-36{ }^{\circ} \mathrm{C}$. The temperature at which clear, flowable microgel phase and swollen microgels suspension phase co-exist is called gelling temperature (GT). Flowable characteristic of microgel was in fact due to weakening of hydrogen bonding present between water molecules and microgel functionalities in result of increase in temperature. Further increase of temperature in range of 36$40{ }^{\circ} \mathrm{C}$ increased the strength of polymer-polymer hydrogen bonding as compared to polymer-water hydrogen bonding. Also, hydrophobic interactions were established between polymer chains and clear suspension turned into milky appearance. The temperature at which this phase transition was occurred is called cloud point temperature (CPT). Above $40{ }^{\circ} \mathrm{C}$, microgel suspension was turned into shrunken state rapidly. At this stage, water come out of microgels network. This temperature is called VPTT. Further increase in temperature may cause aggregation of microgel particles. Crosslinker content of microgels also tune their phase transition behavior.

\subsection{Phase transition kinetics of P(NIPAM-Am) based microgels}

Phase transition kinetics of P(NIPAM-Am) microgels was studied by Wang et al. using UV-visible spectroscopy ${ }^{\mathbf{1}}$ which is an excellent tool for this purpose. ${ }^{50}$ The value of transmittance of the microgel samples containing different Am contents measured at their VPTT was found to be decreased with time and became constant at deswelling equilibrium. The time required to attain deswelling equilibrium was increased with increase of Am contents. As Am is hydrophilic in nature and its high contents inside the polymer network interacted with large amount of water through hydrogen bonding. Thus, more time is required to expel out water from microgel to attain deswelling equilibrium. Similarly swelling kinetics of the microgel samples was studied by measuring value of transmittance at $12{ }^{\circ} \mathrm{C}$ as a function of time. The value of time required to attain swelling equilibrium was found to be decreased with increase of Am contents in the microgels. They reported that concentration of P(NIPAM-Am) microgel particles also affects the swelling/ deswelling kinetics of dispersion. Deswelling equilibrium of microgel dispersion with its high concentration was attained in small time (240 s) as compared to that for microgel dispersion 
with low concentration (300 s). While swelling equilibrium was attained more rapidly in microgel dispersion with low concentration as compared to microgel dispersion with high concentration.

\section{Applications of P(NIPAM-Am) based microgels}

\subsection{P(NIPAM-Am) based microgels for stabilization of inorganic nanoparticles}

Polymer microgels containing NIPAM and Am have gained much attention as micro-reactors for in situ fabrication of inorganic nanoparticles in the last two decades due to potential applications of resulting hybrid microgels. ${ }^{5,9,10,23,26,36,65}$ Deep literature review reveals that NIPAM and Am based microgels containing some additional ionic moiety are widely reported for this purpose ${ }^{5,66}$ but synthesis of metal nanoparticles within $\mathrm{P}$ (NIPAM-Am) microgels has been rarely reported in literature. We are the first who reported fabrication and stabilization of silver nanoparticles in this particular microgel system using in situ reduction of silver nitrate $\left(\mathrm{AgNO}_{3}\right)$ by $\mathrm{NaBH}_{4} \cdot{ }^{5}$ There is no straight forward report on fabrication of metal nanoparticles within P(NIPAM-Am) microgel dispersion before our work. However polymerization/crosslinking of NIPAM and Am around a single gold nanoparticle using $N, N$-methylene bis acrylamide as crosslinker, APS as initiator and SDS as emulsifier has been reported. ${ }^{67}$ In this method, Au nanoparticles were synthesized using citrate reduction of chloroauric acid. The surface of $\mathrm{Au}$ nanoparticles was modified by growing a monolayer of Am around $\mathrm{Au}$ nanoparticles. Then NIPAM and Am were polymerized around $\mathrm{Au}$ nanoparticles by free radical precipitation polymerization as described in Section 2 to obtain Au@IPN$\mathrm{P}($ NIPAM-Am) core shell hybrid microgels with $\mathrm{Au}$ single nanoparticle core and P(NIPAM-Am) shell. The central core made of Au nanoparticles is surrounded by polymeric shell and cannot be accessed easily due to which its applications become limited. Another alternate approach of fabrication of hybrid microgel system is stabilization of metal nanoparticles on the surface of microgel particle. These particles are easily accessible due to their presence on the surface of polymer microgel particle. Fang et al. synthesized $\mathrm{Fe}_{3} \mathrm{O}_{4} @ \mathrm{P}(\mathrm{NIPAM}-\mathrm{Am}$ ) core shell composite particles with ferric oxide $\left(\mathrm{Fe}_{3} \mathrm{O}_{4}\right)$ nanoparticle core and $\mathrm{P}(\mathrm{NIPAM}-\mathrm{Am})$ shell and attached gold nanoparticles to the surface of $\mathrm{Fe}_{3} \mathrm{O}_{4} @ \mathrm{P}(\mathrm{NIPAM}-\mathrm{Am})$ core shell composite particles to obtain magnetic hybrid microgels. ${ }^{8}$ But metal nanoparticles present on the surface of polymer microgel particles are not stable enough for their potential applications. So the best strategy to obtain stable and applied hybrid microgels is fabrication of metal nanoparticles within microgels. The metal nanoparticles cannot only be fabricated in microgels but also can be stabilized for a long time due to some sort of interaction between them and functional groups of the polymeric network. $\mathrm{P}$ (NIPAM-Am) microgels may be a potential candidate for stabilization of metal nanoparticles due to interaction between amide groups and metal nanoparticles but stabilization of metal nanoparticles in these microgels has not been widely reported in this particular system as compared other microgels. Such type of hybrid microgels can be used as catalysts for various organic transformations because acrylamide moiety is the most inert one and does not interfere with organic species participating or producing in catalyzed organic reactions. The content and stability of metal nanoparticles within polymer network can be enhanced by their fabrication in P(NIPAM-AmIM) microgels instead of P(NIPAM-Am) microgels. Therefore a lot of work on fabrication of metal nanoparticles within $\mathrm{P}$ (NIPAM-Am-IM) polymer microgels for various applications has been reported in modern literature..$^{\mathbf{9 1 4 , 1 7 , 2 6 , 6 5}}$

\subsection{Use of $P($ NIPAM-Am) based microgels loaded with metal nanoparticles in surface enhanced Raman scattering}

Noble metal nanoparticles stabilized by P(NIPAM-Am) microgels can be used in surface enhanced Raman scattering. Fang et al. used $\mathrm{Fe}_{3} \mathrm{O}_{4} @ \mathrm{P}(\mathrm{NIPAM}-\mathrm{Am}) @ A u$ hybrid microgels for this purpose. $^{8}$ They recorded Raman spectra of $1 \times 10^{-6} \mathrm{M}$ crystal violet (CV) with and without of $\mathrm{Fe}_{3} \mathrm{O}_{4} @ \mathrm{P}(\mathrm{NIPAM}-\mathrm{Am}) @ \mathrm{Au}$ hybrid microgels. A significant enhancement in intensity of the signals was observed in the presence of hybrid microgels. Thus noble metal nanoparticles fabricated P(NIPAM-Am) microgels are employed in surface enhanced Raman scattering.

\subsection{Use of $\mathbf{P}(\mathrm{NIPAM}-\mathrm{Am})$ based microgels in etalons}

Smart polymer microgels have been widely used in photonics particularly in etalons. ${ }^{\mathbf{1 5 , 6 8 - 7 1}}$ Etalons are modern interferometers. A dielectric medium is sandwiched between two reflecting surfaces in an etalon. ${ }^{15} \mathrm{P}(\mathrm{NIPAM}-\mathrm{Am})$ microgels acts as dielectric layer while metal nanoparticles film acts as reflecting surface in an etalon. If a light beam is incident upon an etalon, then it will be reflected at dielectric-reflecting interface many times as a result of which reflectance spectrum is obtained whose properties can be defined by the following equation

$$
m \lambda_{\max }=2 n d \cos \theta
$$

where $m$ is peak order, $\lambda_{\max }$ is wavelength associated to maximum reflectance, $d$ is spacing between reflecting surfaces, $n$ is refractive index of dielectric medium and $\theta$ is angle of incidence. This relation has been found valid for microgels based etalon. Microgels based etalons are more useful than that of other materials due to following reasons. Microgels possess responsive nature, so the spacing (thickness) and refractive index of the dielectric of etalon can be easily controlled by external stimuli like $\mathrm{pH} /$ temperature. On the other hand, it is difficult to change the thickness of etalon having nonresponsive dielectric because if an undesired thickness of etalon has been built then it is of no use further. One has to rebuild the etalon for work. Microgels are elastic materials and contained large amount of water within their network. While other materials like graphite etc. are solid and possess high toughness. Therefore chances of scattering are very low in case of microgels based etalon as compared to that of other materials.

Sorrell et al. had proved the validity of this relation (eqn (1)) for a variety of etalons in which P(NIPAM-AA), P(NIPAM-Am) 
and P(NIPAM-VAA) microgels had been used as dielectric medium. ${ }^{15}$ They determined the effect of spacing $(d)$, order $(m)$ and refractive index $(n)$ on the value of $\lambda_{\max }$. They also proved that value of $\lambda_{\max }$ was decreased with decrease in spacing. They increased the temperature of P(NIPAM-Am), P(NIPAM-AA) and $\mathrm{P}$ (NIPAM-VAA) microgels based etalon from 23 to $39{ }^{\circ} \mathrm{C}$ to decrease the size of microgel particle, so that the spacing of etalon would decreased resultantly. They also observed that value of $\lambda_{\max }$ and size of microgel particles both decreased sigmoidally with increase in temperature. It means a desired value of $\lambda_{\max }$ can be achieved by adjusting the value of $m, n$, $d$ and $\theta$. Sorrell et al. had prepared three microgel samples using different co-monomers. ${ }^{15}$ So their elasticity and refractive index are different from each other. But Sorrel et al. did not correlate the elasticity/refractive index of all the three microgels with $\lambda_{\max }{ }^{15}$ Moreover they synthesized $\mathrm{pH}$ responsive microgel samples [P(NIPAM-AA) and P(NIPAM-VAA)] but they did not study the effect of $\mathrm{pH}$ on $\lambda_{\text {max }}$. However $\mathrm{pH}$ stimulus could be used to modulate the spacing of etalon. Moreover they had carried out whole study at $\mathrm{pH} 4$ at which $\mathrm{pH}$ responsive microgel samples are in deswollen state and carry no charge. The reflectance spectra should also be investigated under basic conditions ( $\sim \mathrm{pH}$ greater than $\mathrm{p} K_{\mathrm{a}}$ of AA and VAA).

\subsection{Use of P(NIPAM-Am) based polymer microgels in ordered surface structures}

The use of microgel particle self-assemblies to generate periodic arrays (2D or $3 \mathrm{D}$ ) onto some substrate like silicon wafer or metal film has gained a lot of attention due to their potential use in photonic, nano-fluidic and nano-lithography. ${ }^{18,36}$ To obtain periodic loosely packed (PLP) assemblies, a thin homogenous layer of microgel particles is coated on a substrate like silicon, metal or glass film. Then the microgel coating can be dried by free evaporation or droplet drying methods. The microgel coated surface is exposed to air at room temperature to dry the microgel layer in free evaporation method. While microgel coated surface is exposed to a hot flux of air for 10-15 seconds in droplet drying method to dry the microgel layer. Droplet drying method is more efficient as compared to free evaporation method. Free evaporation method took days while droplet drying method did same job within seconds. Moreover organized arrays of microgel particles on substrate are not formed by free evaporation method while completely organized arrays of microgel particles are formed by droplet drying method. That's why scientists prefer droplet drying method over conventional free evaporation method for this purpose. Angle of air flux and distance of air source from substrate can affect the arrangement of microgel particles on substrate. It has been studied that both of these parameters had little or no influence of microgel coating if drying is completed within 10-15 seconds. It has been also reported that closeness of microgel arrays only depends upon size of microgel particles. The arrangement of microgels particles is independent of their charge density, composition and sieve size. Horecha et al. has reported the aforementioned findings. ${ }^{36}$ They synthesized three silicon surfaces coated with P(NIPAM-Am), P(NIPAM-AA) and
P(NIPAM-VAm) microgels. These samples possess different charge densities. But they studied that long range ordered arrays were formed by all samples which depicted that order of arrays did not depend upon charge. They also made porous coatings with the help of microgels. They sputtered microgel coated silicon wafer with nickel and gold films. Then they removed microgel layer by ultra-sonication. Thus pores were developed at place of microgel in nickel/gold films. They confirmed the formation of pores using SEM and AFM images.

\subsection{Enzymes immobilization with tunable activity on modified P(NIPAM-Am) based microgels}

Microgels possessing primary amine group $\left(\mathrm{NH}_{2}\right)$ as pendant group are used as platform for immobilization of polymers like proteins and dyes. Recently three approaches have been used to synthesize primary amine terminated microgels: (1) postpolymerization Hoffmann rearrangement, (2) postpolymerization grafting of primary amine groups, and (2) copolymerization of thermo-responsive monomers with cationic co-monomers. Series of microgels with same crosslinking, hydrodynamic diameter and other morphological parameters cannot be achieved through approach (2) and (3). Therefore approach $\mathrm{I}$ is considered better than other two approaches. $\mathrm{P}$ (NIPAM-Am) microgels possess amide group as terminal group. These amide groups can be successfully converted into amine groups through Hoffmann rearrangement. Shiroya et al. has synthesized aminated microgels by Hoffmann rearrangement and used them as platform for immobilization of trypsin enzyme. ${ }^{72}$ Initially they produced terminal amine groups on P(NIPAM-Am) microgels by Hoffman rearrangement. All the amide groups were not converted into amine groups by Hoffmann rearrangement. Then they oxidized the remaining amide groups into carboxyl groups by base hydrolysis. In this way they synthesized P(NIPAM-Am)-mod microgels which possess amine and carboxyl both as terminal groups. Then they immobilized the trypsin enzyme within microgels by carbodiimide coupling. Then they modulated the activity of enzyme by thermoresponsive swelling/deswelling of microgels. VPTT of $\mathrm{P}$ (NIPAM-Am) modified microgels was $33{ }^{\circ} \mathrm{C}$ while VPTT of trypsin-P(NIPAM-Am) modified microgels was $\sim 40{ }^{\circ} \mathrm{C}$. They immobilized 2.2 and $4.1 \mathrm{mg}$ trypsin in $1 \mathrm{~g}$ of microgels then they observed that enzymatic activity was decreased when temperature was increased above VPTT of microgels. Actually microgels got deswell with increase in temperature, so the diffusion of substrate molecules was decreased and enzymatic activity decreased resultantly. They also observed that enzymatic activity was decreased gradually when VPTT was approached while $R_{\mathrm{h}}$ of microgels was decreased sharply at that temperature. These results were indicated that some enzyme molecules present at peripheral region of microgel moved onto surface of microgel particles due to deswelling as shown in Fig. 4. So the enzymatic activity of microgels did not shut off completely. Enzyme molecules present at surface of microgels catalyzes the process. That's why the trypsin activity was decreased gradually around VPTT. Shiroya et al. has converted amide into amine group but it is clear from their results that all the amide groups 


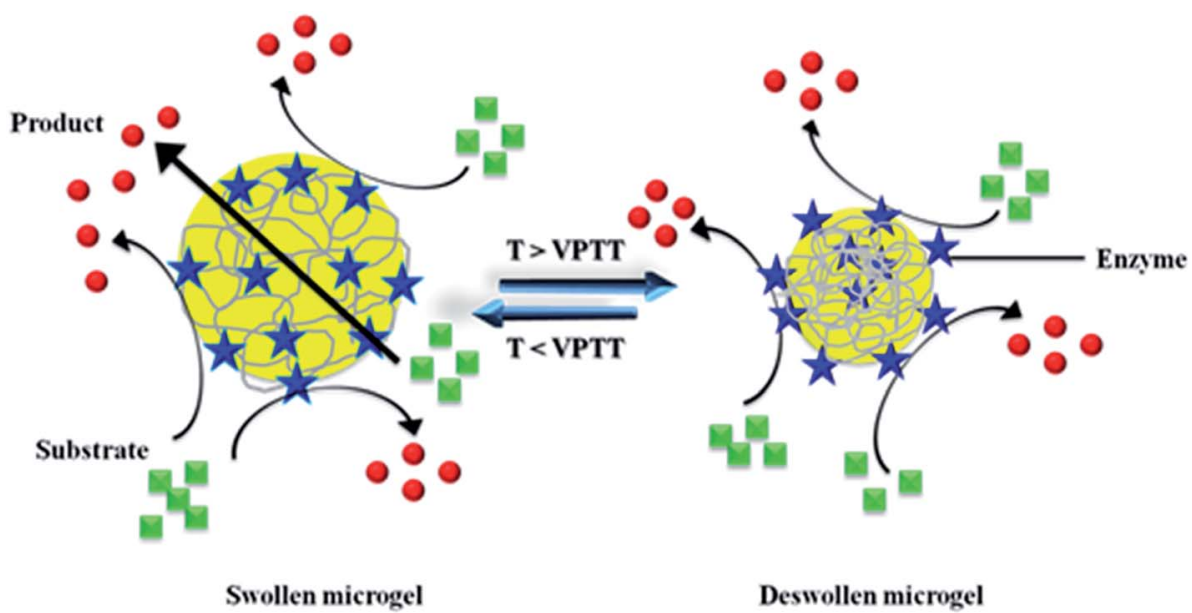

Fig. 4 Catalytic conversion of substrate into product by enzyme molecules mobilized in responsive microgel at different temperatures

had not been converted into amine groups by Hoffmann rearrangement. ${ }^{72}$ They did not study the $\mathrm{pH}$ responsive behavior of $\mathrm{P}$ (NIPAM-Am) modified microgels which might reveal the amphoteric nature of these microgels because amine and carboxyl both groups were present within same particle. They carried out complete study at $\mathrm{pH} 7$, but they were not aware that either particle is in maximum swollen state here or not. Because VPT $\mathrm{pH}$ of amphoteric particles depends upon $\left[\mathrm{NH}_{2}\right] /[\mathrm{COOH}]$ ratio. As they did not report the number of amine and carboxyl groups in P(NIPAM-Am) modified microgels, so their study is not comprehensive.

Later Wang et al. reported all these parameters comprehensively. ${ }^{35}$ They discovered that all amide groups can be converted into amine groups completely if NIPAM was replaced by NIPMAM and Am was replaced by MAAm. They also reported that Hoffmann side reaction also occurred along with main reaction when $\mathrm{P}$ (NIPAM-Am) microgels were treated with bleach (reagent for Hoffmann reaction). ${ }^{35}$ Wang et al. also pointed out that crosslinker BIS hydrolyzed in the presence of bleach. ${ }^{8}$ Wang et al. had overcame these drawbacks by replacing $\mathrm{P}(\mathrm{NIPAM}-\mathrm{Am})$ microgels with poly( $N$-isopropylmethacrylamidemethacrylamide) [P(NIPMAM-MAm)] as platform for enzymes immobilization. ${ }^{35}$ They also replaced crosslinker BIS with ethylene glycol dimethacrylate (EGDM). They prepared four different microgels: P(NIPAM-Am), P(NIPMAM-MAm), poly $(N$ isopropylmethacrylamide-methacrylamide-acrylic [P(NIPMAM-MAm-AA)] and poly $(N$-isopropylmethacrylamide $)$ [P(NIPMAM)]. P(NIPMAM-MAm), P(NIPMAM-MAm-AA) and $\mathrm{P}$ (NIPMAM) microgels were crosslinked by EGGM while $\mathrm{P}$ (NIPAM-Am) microgels were crosslinked by BIS. They treated all microgels with bleach in basic media. They found that crosslinker EDGM did not hydrolyze while crosslinker BIS hydrolyze in basic media. They treated P(NIPMAM-MAm) and $\mathrm{P}$ (NIPAM-Am) microgels with bleach and investigated their electrophoretic mobility. They found that P(NIPMAM-MAm) microgel sample did not give negative potential value at any $\mathrm{pH}$ value. While P(NIPAM-Am) microgels sample showed negative potential value after $\mathrm{pH} 8.5$ (isoelectric point $=8.5 \mathrm{pH}$ ).
This showed that Hoffman side reaction did not occur when P(NIPMAM-MAm) microgels was treated with bleach. They also investigated that $51 \%$ of amide groups were converted into amine groups during Hoffman rearrangement. They also studied the $\mathrm{pH}$ responsive behavior of samples after Hoffmann rearrangement. They measured the $R_{\mathrm{h}}$ values of particles using DLS instrument at different $\mathrm{pH}$ values of medium. They observed that $R_{\mathrm{h}}$ value of NIPMAM based sample was decreased with increase in $\mathrm{pH}$ of media. They also observed that $R_{\mathrm{h}}$ of NIPAM based sample was decreased with increase in $\mathrm{pH}$ up to $\mathrm{pH}=8.5$, when $\mathrm{pH}$ of medium was increased above 8.5 then $R_{\mathrm{h}}$ value of particles was started to increase. It meant, NIPMAM based sample showed basic behavior while NIPAM based sample showed amphoteric behavior. This result also confirmed that carboxyl groups were formed in NIPAM based sample only. They investigated that amount of amine groups formed depends upon time. Thus they prepared a series of amine terminated microgels by varying the time of reaction. They prepared samples by carrying the reaction for 10, 30 and $60 \mathrm{~min}$. They found that $13 \%, 26 \%$ and $51 \%$ of the amide groups were converted into amine groups at 10, 30 and $60 \mathrm{~min}$ in P(NIPMAM-MAAm) sample respectively. They also treated $\mathrm{P}$ (NIPMAM-MAAm-AA) microgels with bleach for various time. Thus they prepared a series of samples having $\left[\mathrm{NH}_{2}\right] /[\mathrm{COOH}]$ ratio $0.4,0.6$ and 1.4 with isoelectric point at $\mathrm{pH} 4,4.5$ and 6.8 respectively. In this way they prepared series of amine terminated microgels of P(NIPMAM-MAm) and P(NIPMAMMAm-AA) samples. Samples of these two series possess different number of amine groups only while morphological characters, $R_{\mathrm{h}}$, crosslinking density and monomers distribution in polymer were same. These attributes can be achieved in amine terminated microgels prepared through postpolymerization grafting and copolymerization with cationic co-monomers. They also treated all microgel samples with fluorescein isothiocyanate dye. They compared the UV-visible spectra of all samples and concluded that dye was attached to the microgel having amine group only. Because spectra of amine terminated microgel sample showed peak only while 
spectra of all other samples having no amine group showed no peak. Thus they practically studied the working of amine terminated microgels.

\subsection{Use of P(NIPAM-Am) based microgels in catalysis}

NiPAm and Am with some ionic co-monomer based hybrid microgels have also been extensively used as catalysts in different reactions due to high surface-to-volume ratio of inorganic nanoparticles loaded into the polymeric network. ${ }^{4,13,20,26,27,42}$ P(NIPAM-AAm) microgels may be an ideal carrier for nanoparticles to be used for catalysis due to inertness of functionalities of polymeric network. Pendant isopropyl and amide groups of NIPAM and Am units keep the nanoparticles fix inside the network without any aggregation. Crosslinking of network act as physical barrier against coalescence of nanoparticles. Moreover Am is hydrophilic so it increases the water holding capacity of network. So Am makes the catalyst more suitable for catalysis to be carried out in aqueous medium. Responsive microgels have ability to swell/deswell reversibly in response to various stimuli. The catalytic activity of nanoparticles fabricated within microgels can be controlled by swelling/deswelling of microgel network. Temperature is the reported trigger for swelling/deswelling of P(NIPAM-Am) hybrid microgels. ${ }^{26,73}$ Another important feature of P(NIPAM-Am) hybrid microgels is that the catalytic activity of the hybrid system can be tuned by varying temperature of the medium because NIPAM moiety of hybrid microgels becomes hydrophobic at high temperature as a result of which polymer network deswells. The shrinkage of the network creates a barrier in diffusion of reactant molecules towards the catalyst surface due to which rate of reaction decreases. Similarly polymer network swells at low temperature and allows the diffusion of reactants towards surface of nanoparticles to enhance the rate of reaction. On the other hand, amide pendant groups are present in close vicinity of nanoparticles in deswollen state, so they block the active sites of nanoparticles. Ajmal et al. have reported temperature and $\mathrm{pH}$ as triggers (stimuli) to control the catalytic activity of Ag-P(NIPAM-Am-MAA) hybrid microgels. ${ }^{26}$ They used NIPAM and MAA to make the microgels temperature and $\mathrm{pH}$ responsive at the same time. Catalytic reduction of 4nitrophenol (4-NP) was carried out in basic medium. Carboxyl group of MAA is ionized at high $\mathrm{pH}\left(\mathrm{pH}>\mathrm{p} K_{\mathrm{a}}\right)$ and converted into negatively charged carboxylate ions which makes the carrier swollen and hydrophilic in basic medium. They investigated the catalytic reduction at 25 and $55^{\circ} \mathrm{C}$. They found $12 \times$ $10^{-2}$ and $41 \times 10^{-2} \mathrm{~min}^{-1}$ as value of apparent rate constant at 25 and $55{ }^{\circ} \mathrm{C}$ respectively. They also studied the temperature responsive behavior of microgels at various $\mathrm{pH}$ values. This study revealed that microgels are present in deswollen state at $55^{\circ} \mathrm{C}$ and swollen state at $25^{\circ} \mathrm{C}$. So the value of rate constant at $55^{\circ} \mathrm{C}$ should be smaller than that at $25^{\circ} \mathrm{C}$. But it was observed that the value of rate constant at $55{ }^{\circ} \mathrm{C}$ is greater than that of $25{ }^{\circ} \mathrm{C}$. This is due to the Arrhenius behavior of reaction. They did not report the catalytic reduction of 4-NP at various temperatures, hence the effect of temperature on rate constant cannot be understood clearly from this study. Wu et al. have reported glucose as trigger for catalytic activity of $\mathrm{Au}$ nanoparticles. ${ }^{73}$ They synthesized glucose responsive Au-P(NIPAMAm-VPBA) core-shell hybrid microgels. They studied effect of hydrophilic/hydrophobic state of microgel on the catalysis of 4NP and nitrobenzene in aqueous medium. They studied that hybrid microgels are glucose responsive in $0-4 \mathrm{mM}$ glucose concentration range only. They investigated that size of hybrid microgel increases with increase in glucose concentration up to $4 \mathrm{mM}$. While size of hybrid microgel was very slightly increased when glucose concentration was increased from 4 to $5 \mathrm{mM}$. They studied catalytic reduction of 4-NP and NB in $0-5 \mathrm{mM}$ glucose concentration range. They concluded that value of apparent rate constant for reduction of 4-NP increases with increase in glucose concentration while value of $k_{\text {app }}$ for reduction of NB decreases with increase in glucose concentration. Actually microgels are hydrophilic in swollen state, so they allow the diffusion of hydrophilic 4-NP and hinder the diffusion of hydrophobic NB and vice versa. That is why glucose concentration dependence of $k_{\text {app }}$ of 4-NP reduction was found to be different from that of $k_{\text {app }}$ of NB reduction. Thermally tunable catalytic reduction of 4-nitroanline using P(NIPAM-Am-MAA) hybrid microgels has been recently reported by our group. ${ }^{24}$

We are the pioneer who reported the use of Ag nanoparticles fabricated P(NIPAM-Am) hybrid microgels as catalyst for reduction of 4-nitrophenol in aqueous medium. ${ }^{13}$ Reduction of 4-NP was carried out at ambient temperature using sodium borohydride as reducing agent in presence of Ag-P(NIPAM-Am) hybrid microgels catalysts under various reaction conditions in aqueous medium. Our detailed studies on catalytic activity of NIPAM and Am based hybrid microgels can be found in literature. ${ }^{13,26,27}$ Catalytic activity of P(NIPAM-Am) based hybrid microgels has not been extensively explored in literature which stimulate us to carryout work on synthesis, characterization and catalytic applications of metal nanoparticles in P(NIPAM-Am) microgels for various organic reactions. Some work on this particular system has been already reported by us and more extensive study on it is in progress in our laboratory and will be published shortly.

\subsection{Optical sensing applications of acrylamide based microgels}

$\mathrm{P}(\mathrm{NIPAM}-\mathrm{Am})$ microgels and P(NIPAM-Am-AA) microgels have a potential to be used as templates for immobilization of assemblies of azo dyes for optical sensing applications. $\mathrm{Wu}$ et al. ${ }^{59}$ used P(NIPAM-Am) microgels and P(NIPAM-Am-AA) microgels as support for loading of assembly of Calcon dye molecules. P(NIPAM-Am)-Calcon composite microgels $\mathrm{P}$ (NIPAM-Am-AA)-Calcon composite microgels were obtained by adding Calcon dye aqueous solution into pure microgels dispersion dropwise followed by two days stirring under $\mathrm{N}_{2}$ atmosphere and acidic $\mathrm{pH}$ conditions at room temperature. The composite microgels were purified by dialysis of the dispersion against distilled water. Absorption band of free Calcon solution is shifted towards longer wavelength with increase in $\mathrm{pH}$ but this shift is not systematic. Moreover a new band around $636 \mathrm{~nm}$ wavelength is found to appear in highly basic 
conditions. The absorption band of composite microgels is systematically shifted towards longer wavelength with increase in $\mathrm{pH}$ of medium and number of bands is same under acidic and basic $\mathrm{pH}$ conditions. The $\mathrm{pH}$ of medium is affected by concentration of $\mathrm{H}_{2} \mathrm{O}_{2}$. So the concentration of $\mathrm{H}_{2} \mathrm{O}_{2}$ can be easily sensed by the position of absorption band of composite microgels. In this way composite microgels play crucial role in optical sensing of $\mathrm{H}_{2} \mathrm{O}_{2}$.

Optical glucose sensing of NIPAM and Am based polymer microgels loaded with inorganic nanoparticles has been a subject of interest for a wide community of researchers. ${ }^{74,75}$ Biocompatible and fluorescent carbon dots (CDs) with average diameter of $6 \mathrm{~nm}$ loaded in glucose-imprinted poly $(N$ isopropylacrylamide-acrylamide-vinylphenylboronic acid) [P(NIPAM-AAm-VPBA)] polymer microgels can be used for optical detection of glucose under physiological conditions. ${ }^{76}$ The variation in glucose concentration can cause quenching in CDs signals in a reversible way and can be measured easily. The variation in intensity of fluorescent signal with variation of glucose concentration of surrounding medium is attributed to its complexation with PBA which results in swelling of polymeric network.

\subsection{Biomedical applications of P(NIPAM-Am) based microgels}

Smart polymeric material has gained a great deal of attention in biomedical applications and a flood of publications dealing with this area has been appeared in literature ${ }^{77-88}$ but our discussion is limited to biomedical applications of P(NIPAMAm) and P(NIPAM-Am-IM) polymer microgels and their hybrids as given below.

5.8.1. Thermotherapy or tumor hyperthermia. Thermotherapy is the controlled use of heat to treat cancer. Magnetic nanoparticles fabricated P(NIPAM-Am) microgels can be used to treat cancer cells. $\mathrm{Fe}_{3} \mathrm{O}_{4}-\mathrm{P}(\mathrm{NIPAM}-\mathrm{Am})-\mathrm{Au}$ hybrid microgels have been employed for this purpose. ${ }^{8}$ Temperature of the system increases with increase of exposure time of AC magnetic field over hybrid microgels as shown in Table 2 . Temperature required in thermotherapy is $42-46{ }^{\circ} \mathrm{C}$. Thus, $\mathrm{Fe}_{3} \mathrm{O}_{4}-\mathrm{P}$ (NIPAM-Am)-Au hybrid microgel can be used for destruction of tumor cells.

There is another way through which $\mathrm{P}$ (NIPAM-Am) microgels can also be used for biomedical applications. P(NIPAM-Am)

Table 2 Magneto caloric effect of $\mathrm{Fe}_{3} \mathrm{O}_{4}-\mathrm{P}(\mathrm{NIPAM}-\mathrm{Am})-\mathrm{Au}$ hybrid microgels

\begin{tabular}{ll}
\hline Time $(\mathrm{s})$ & Temperature $\left({ }^{\circ} \mathrm{C}\right)$ \\
\hline 65 & 27.5 \\
150 & 32.0 \\
225 & 35.0 \\
300 & 37.5 \\
375 & 40.0 \\
450 & 42.5 \\
525 & 43.5 \\
600 & 44.2 \\
675 & 44.7 \\
750 & 45.0
\end{tabular}

hybrid microgels can be loaded with Plasmonic nanoparticles which have tendency to absorb light radiations and convert this absorbed light energy into heat. Gold or silver nanoparticles possess SPR wavelength in visible range. It has been observed if radiation of SPR wavelength of nanoparticles is bombarded on hybrid microgel as a result microgels got deswell. This is termed as photo-thermal deswelling. Actually nanoparticles transform the light energy into heat energy and transfer this heat energy to microgels. So the temperature of microgels is increased and deswelling occurred. Shiotani et al. have studied the effect of photo-thermal deswelling on the bio-distribution of microgels within mice body ${ }^{25}$ (Fig. 5). They synthesized Au nanorods coated with poly( $N$-isopropylacrylamide- $N, N$-dimethylacrylamide) [P(NIPAM-DMAm)] and P(NIPAM-Am) microgels having VPTT around 37 and $45^{\circ} \mathrm{C}$ respectively in aqueous medium. So $\mathrm{Au}-\mathrm{P}(\mathrm{NIPAM}-\mathrm{DMAm})$ microgels got deswell and Au-P(NIPAM$\mathrm{Am})$ did not deswell when injected into mice. That is why $\mathrm{Au}-$ $\mathrm{P}$ (NIPAM-Am) hybrid microgels are more suitable for photothermal treatment of tumors than Au-P(NIPAM-DMAm) hybrid microgels. They injected Au-P(NIPAM-Am) hybrid microgels into body and analyzed the \% injection dose/gram of tissue in various body parts after $30 \mathrm{~min}$ and $72 \mathrm{~h}$. They observed that maximum \% injection dose/gram of tissue were present in blood and spleen after $30 \mathrm{~min}$ and in spleen after 72 hour of injection. They also irradiated the right tumor with near infra-red laser of intensity $3.8 \mathrm{~W} \mathrm{~m}^{-2}$ for $10 \mathrm{~min}$ but they did not irradiate the left tumor. They compared the \% injection dose/ gram of tissue of left and right tumor. They analyzed that hybrid microgels accumulated in right tumor was greater than that of left tumor. They explained it on the basis of enhanced permeability and retention (EPR) effect. As the right tumor was irradiated, so the hybrid microgels were in de-swollen and hydrophobic state in this tumor. So they got accumulated here in the fine capillaries, cellular spaces of vessels and extracellular matrix due to the hydrophobic interactions and very small size of hybrid microgels. They did not optimize various parameters like how much injection dose is sufficient, does surface structure of hybrid microgels suitable or not, etc. Their work was of basic nature. A lot of work is needed in this area. Scientists had reported the effect of photo-thermal treatment on tumor cells like Zhang et al. had studied the photo-thermal treatment of tumor cell in mice by Au-P(NIPAM-AA) hybrid microgels. ${ }^{89}$ But $\mathrm{Au}-\mathrm{P}(\mathrm{NIPAM}-\mathrm{AA})$ hybrid microgels are not neutral. They possess ionic co-monomer AA whose $\mathrm{p} K_{\mathrm{a}}$ value is $\sim 4.5$. The $\mathrm{p} K_{\mathrm{a}}$ value of Ac is less than $\mathrm{pH}$ of blood $\sim 7.3$. So Au-P(NIPAM-AA) microgels were converted into swollen and negatively charged state when injected into body. So Au-P(NIPAM-AA) microgels would not deswell easily on laser irradiation in comparison to $\mathrm{Au}-$ $\mathrm{P}$ (NIPAM-Am) microgels. Chemotherapy is an alternate methodology which is widely used for treatment of a variety of cancers. Cancer treatment efficiency can be significantly enhanced by combining both therapies in a single system. P(NIPAM-Am) based Chemo-photothermal therapy has been recently introduced. ${ }^{90} \mathrm{P}(\mathrm{NIPAM}-\mathrm{Am})$ polymer nanogel particles were fabricated within a liposome template to obtain core shell system with P(NIPAM-Am) thermosensitive core and liposome shell. An anticancer drug doxorubicin hydrochloride and 


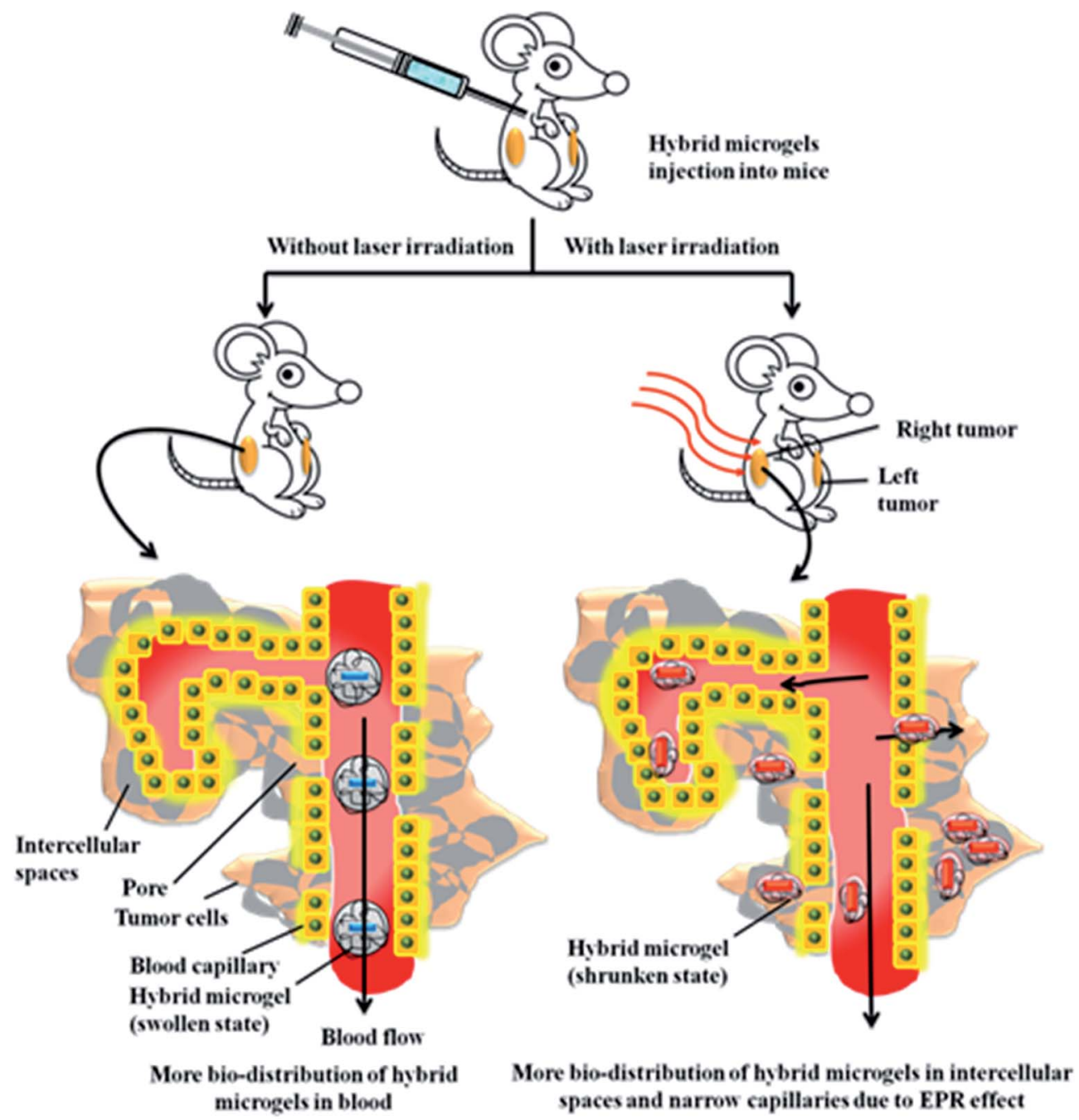

Fig. 5 Distribution of hybrid microgels in mice in the presence/absence of laser irradiation.

a photosensitizer (indocyanine green) both were encapsulated within P(NIPAM-Am) core by ammonium sulfate gradient methodology. Upon irradiation, Indocyanine green converts light energy into heat energy to create hyperthermia and to induce deswelling in polymer core simultaneously which results in quick release of anticancer drug to tumor cells. The presence of liposome layer around P(NIPAM-Am) nanogel particle may cause barrier in release of anticancer drug in above described system. However rate of release of drug from P(NIPAM-Am) can be increased by changing the morphology of P(NIPAM-Am) based anticancer drug delivery system. For example $\mathrm{SiO}_{2}$ core containing $\mathrm{ZnS}: \mathrm{Mn}^{2+}$ and $\mathrm{Fe}_{2} \mathrm{O}_{3}$ quantum dots can be covered by $\mathrm{P}(\mathrm{NIPAM}-\mathrm{Am})$ shell to design more facile anticancer drug release system as reported by Cao et al. ${ }^{91}$

Cytotoxicity of microgels must be studied before in vivo application of microgels. Wu et al. ${ }^{14}$ has reported the cytotoxicity of P(NIPAM-Am) microgels. They synthesized Ag nanoparticles of diameter $40 \mathrm{~nm}$ loaded them into poly $\left(N^{-}\right.$ isopropylacrylamide-acrylamide-4-vinyl phenyl boronic acid) [P(NIPAM-Am-VPBA)] microgels. They studied the viability of B16F10 liver cells in the presence of hybrid microgels and naked
Ag nanoparticles. It is well known fact that silver nanoparticles possess cytotoxic nature. They observed that cells were remained viable $100 \%$ in the presence of hybrid microgels but the viability of cells was decreased in the presence of naked nanoparticles. So it was concluded that cytotoxic nature of silver nanoparticles has been masked by the microgel network. Their results very well support that hybrid microgels can be successfully used for the in vivo drug delivery and bio-sensing.

5.8.2. Stimuli responsive drug release from acrylamide based microgels. Acrylamide based temperature responsive colloidal microgels are frequently used for drug delivery. ${ }^{16}$ The microgels loaded with drug molecules can be injected easily to target cells in the form of aqueous suspension as compared to other macroscopic hydrogels. Moreover microgels have quick response as compared to macro gels due to which they have been widely used as drug delivery systems. Different temperature and $\mathrm{pH}$ responsive microgels based on acrylamide moieties have been reported for controlled drug delivery to target cells. ${ }^{11,92}$

Sung et al. reported crosslinked gelatin based microgels loaded with P(NIPAM-Am) polymer chain as biocompatible and 
temperature controlled drug delivery system of bovine serum albumin (BSaN). ${ }^{11}$ They investigated the kinetics of release of $\mathrm{BSaN}$ from aforementioned microgel system under heating and cooling cycles at 22 and $42{ }^{\circ} \mathrm{C}$ and reported that more than $60 \%$ drug can be released from carrier after four heating and cooling cycles within 3 hours while only $10 \%$ release is obtained at constant temperature of $22^{\circ} \mathrm{C}$ in the same time. The increase in drug release with increase in temperature may be attributed to conversion of coil to globule conformation of P(NIPAM-Am) minor component of microgel system. This change in conformation causes reduction in volume of elastic gelatin microgel particles which enhances the convective flow of drug from polymer network to outside. They also reported that biocompatibility and rate of drug release from microgels can be tuned by concentration of P(NIPAM-Am) and extent of crosslinking of gelatin matrix. They claimed that this drug delivery system is biodegradable but in fact P(NIPAM-Am) component is not biodegradable. However they used this component in very small quantity which can be excreted by kidney and can be removed via renal filtration.

Wang et al. ${ }^{12}$ used P(NIPAM-Am) microgels as carrier of water soluble bleomycin which is an anti-cancer drug used for chemotherapy. The microgels were loaded with drug to obtain uniform suspension. Progress of drug release from microgel suspension was monitored by UV-visible spectrophotometry by measuring absorbance at $295 \mathrm{~nm}$. It was concluded that rate of drug release is strongly dependent upon composition and concentration of P(NIPAM-Am) microgel particles. Moreover drug release from microgel system follows Peppas empirical equation and occurs according to Fickian diffusion. They reported that $100 \%$ release of drug from microgel system can be obtained within 25 hours. A rapid delivery of Indomethacin drug from P(NIPAM-Am-HEA) microgel particles with $100 \%$ release within just 100 minutes has been reported by Fundueanu et al. ${ }^{16}$ but this study was carried out in methanol instead of aqueous medium which limits its biomedical practical applications. A lot of many other publications on drug delivery systems based on Nipam and Am are available in scientific literature ${ }^{75,92,93}$ but acrylamide unit of such kind of microgels is toxic and its toxicity has not been properly addressed in this literature. The low level biodegradability and biocompatibility makes these microgels less desired systems for targeted drug delivery because its long term storage in body causes cytotoxicity. More over in aqueous medium, P(NIPAM-Am) microgels aggregate at temperature $>$ LCST and limits their use as drug carrier. However P(NIPAM-Am-IM) microgels are more hydrophilic and may be a better candidate of drug delivery system due to their high stability in a wide temperature range. Therefore $\mathrm{P}$ (NIPAM-Am-IM) microgels have been widely reported as drug delivery systems.

\section{Summary and future perspective}

Poly( $N$-isopropylacrylamide-acrylamide) microgels are important microgels due to their certain characteristics like responsive nature, hydrophilic and inert character as well as their specific volume phase transition temperature that lies around $37^{\circ} \mathrm{C}$. Moreover, in P(NIPAM-Am) microgels, individual properties of NIPAM and Am are maintained. These properties enlarged the usage of these specific microgels in different fields. $\mathrm{P}(\mathrm{NIPAM}-\mathrm{Am})$ microgels have been used in various applications like polymer coating and immobilization, use as micro-reactor for stabilization of nanoparticles, catalysis, sensing of glucose, drug delivery and etalons. The amide group of P(NIPAM-Am) microgels can be converted into amine group successfully and amine terminated microgels can successfully bind polymers (enzymes) and dyes. These microgels are non-cytotoxic at their low concentrations and possess volume phase transition temperature around $37^{\circ} \mathrm{C}$. So they have been used in drug delivery, bio-sensing and photo-thermal treatment. P(NIPAMAm) microgels can develop into a long-range ordered thin layer at metal film, so they have been used in coating. Microgel layer is sandwiched between two metal film layers to made optical device. As the dielectric of the microgel (hydrophobic/ hydrophilic) and distance between two metal plates is controlled by size of microgel, so these microgels are largely used in etalons. So metal nanoparticles fabricated P(NIPAMAm) hybrid microgel can be used in tumor hyperthermia ${ }^{8}$ and spectroscopic analysis. We believe that acrylamide based microgels will gain more and more attention in future due to their special feature of functional colloids. However few aspects of P(NIPAM-Am) and P(NIPAM-Am-IM) polymer microgels have not been properly explored. For example biocompatibility and biodegradability of P(NIPAM-Am) and P(NIPAM-Am-IM) polymer microgels should be studied in detail to practically apply them in biomedical field. Various polymer based materials have been reported for environmental applications to extract/ degrade toxic substances ${ }^{94-104}$ but this aspect of P(NIPAM-Am) and P(NIPAM-Am-IM) microgels has been rarely explored in literature. Silver nanoparticles loaded polymeric material has been extensively reported for antibacterial applications ${ }^{105-107}$ but antibacterial behavior of silver nanoparticles loaded in $\mathrm{P}$ (NIPAM-Am) and P(NIPAM-Am-IM) microgels may be a subject of future studies in this area.

\section{Conflicts of interest}

There are no conflicts to declare.

\section{List of abbreviations}

$\begin{array}{ll}\text { IM } & \text { Ionic moiety } \\ \text { VA } & \text { Vinylamine } \\ \text { LCST } & \text { Lower critical solution temperature } \\ \text { NIPAM } & N \text {-Isopropylacrylamide } \\ \text { NIPMAM } & N \text {-Isopropylmethacrylamide } \\ \text { Am } & \text { Acrylamide } \\ \text { VAA } & \text { Vinyl acetic acid } \\ \text { AA } & \text { Acrylic acid } \\ \text { MAA } & \text { Methacrylic acid } \\ \text { HEA } & \text { Hydroxyethyl acylate }\end{array}$




$\begin{array}{ll}\text { IPN } & \text { Interpenetrating network } \\ \text { PBA } & \text { Phenylboronic acid } \\ \text { VPBA } & \text { 4-Vinyl phenyl boronic acid } \\ \text { VPTT } & \text { Volume phase transition temperature } \\ \text { DMAm } & N, N \text {-Dimethylacrylamide } \\ \text { KPS } & \text { Potassium per sulfate } \\ \text { APS } & \text { Ammonium per sulfate } \\ \text { AiBN } & \text { Azodiisobutyronitrile } \\ \text { MBAAm } & \text { Methylene bis acrylamide } \\ \text { TEMED } & N, N, N^{\prime}, N^{\prime} \text {-Tetramethylethylene-diamine } \\ \text { EGDM } & \text { Ethylene glycol dimethacrylate } \\ \text { NaPS } & \text { Sodium per sulfate } \\ \text { SDS } & \text { Sodium dodecyl sulfate } \\ \text { TEM } & \text { Transmission electron microscopy } \\ \text { DLS } & \text { Dynamic light scattering } \\ \text { UV-vis } & \text { UV-visible spectroscopy } \\ \text { FTIR } & \text { Fourier transform Infrared spectroscopy } \\ \text { SEM } & \text { Scanning electron microscopy } \\ \text { AFM } & \text { Atomic force microscopy } \\ \text { EDX } & \text { Energy dispersive X-ray spectroscopy } \\ \text { RS } & \text { Raman spectroscopy } \\ \text { NMR } & \text { Nuclear magnetic resonance } \\ \text { DMA } & \text { Differential mechanical analysis } \\ \text { LLS } & \text { Laser light scattering } \\ \text { DSC } & \text { Differential scanning calorimetry } \\ \text { TGA } & \text { Thermogravimetric analysis } \\ \text { GT } & \text { Gelling temperature } \\ \text { CPT } & \text { Cloud point temperature } \\ \text { VPBA } & \text { Binyl phenyl boronic acid } \\ \text { BSaN } & \\ & \end{array}$

\section{Acknowledgements}

Authors are grateful to Higher Education Commission Pakistan for financial supports under National Research Program for Universities [No. 20-3995/NRPU/R\&D/HEC/14/1212], Pakistan Program for Collaborative Research (PPCR) [22-3/HEC/R\&D/ PPCR/2018] and University of the Punjab, Lahore, Pakistan under research Grant No:/999/EST.I for the fiscal year of 20172018. Ahmad Irfan extends his appreciation to the Deanship of Scientific Research at King Khalid University for funding through research groups program under grant number R.G.P.2/ $15 / 40$.

\section{References}

1 Q. Wang, Y. Zhao, H. Xu, X. Yang and Y. Yang, J. Appl. Polym. Sci., 2009, 113, 321-326.

2 T. Hoare and R. Pelton, Langmuir, 2004, 20, 2123-2133.

3 R. Pelton, Adv. Colloid Interface Sci., 2000, 85, 1-33.

4 B. M. Budhlall, M. Marquez and O. D. Velev, Langmuir, 2008, 24, 11959-11966.

5 H. Naeem, Z. H. Farooqi, L. A. Shah and M. Siddiq, J. Polym. Res., 2012, 19, 1-10.

6 C. Xiao, Q. Wu, A. Chang, Y. Peng, W. Xu and W. Wu, J. Mater. Chem. A, 2014, 2, 9514-9523.
7 S. Juodkazis, N. Mukai, R. Wakaki, A. Yamaguchi, S. Matsuo and H. Misawa, Nature, 2000, 408, 178.

8 J. Fang, C. Wang, M. Cao, M. Cheng, J. Shi and Y. Jin, Mater. Lett., 2013, 96, 89-92.

9 W. Wu, T. Zhou, M. Aiello and S. Zhou, Biosens. Bioelectron., 2010, 25, 2603-2610.

10 Z. H. Farooqi, A. Khan and M. Siddiq, Polymer Int., 2011, 60, 1481-1486.

11 B. Sung, C. Kim and M.-H. Kim, J. Colloid Interface Sci., 2015, 450, 26-33.

12 Y. Z. Qin Wang, Y. Yang, H. Xu and X. Yang, Colloid Polym. Sci., 2007, 285, 515-521.

13 R. Begum, Z. H. Farooqi, E. Ahmed, K. Naseem, S. Ashraf, A. Sharif and R. Rehan, Appl. Organomet. Chem., 2017, 31.

$14 \mathrm{~W}$. Wu, J. Shen, Y. Li, H. Zhu, P. Banerjee and S. Zhou, Biomaterials, 2012, 33, 7115-7125.

15 C. D. Sorrell and M. J. Serpe, Adv. Mater., 2011, 23, 40884092.

16 G. Fundueanu, M. Constantin, F. Bortolotti, P. Ascenzi, R. Cortesi and E. Menegatti, Macromol. Biosci., 2005, 5, 955-964.

17 K. Khan, A. J. Shaikh, M. Siddiq, T. A. Sherazi and M. Nawaz, J. Polym. Eng., 2016, 36, 287-292.

18 W. Wu, T. Zhou and S. Zhou, Chem. Mater., 2009, 21, 28512861.

19 J. Shen, T. Ye, A. Chang, W. Wu and S. Zhou, Soft Matter, 2012, 8, 12034-12042.

20 Z. Cao, T. Y. Chen, X. L. Guo, X. J. Zhou, J. J. Nie, J. T. Xu and Z. Q. Fan, Chin. J. Polym. Sci., 2011, 29, 439-449.

21 M. Horecha, V. Senkovskyy, K. Schneider, A. Kiriy and M. Stamm, Colloid Polym. Sci., 2011, 289, 603-612.

22 K. Khan, A. J. Shaikh, M. Siddiq, T. A. Sherazi and M. Nawaz, J. Polym. Eng., 2016, 36, 287-292.

23 Y. Li and S. Zhou, Sens. Actuators, B, 2013, 177, 792-799.

24 M. Shahid, Z. H. Farooqi, R. Begum, K. Naseem, M. Ajmal and A. Irfan, Korean J. Chem. Eng., 2018, 1-9.

25 A. Shiotani, Y. Akiyama, T. Kawano, Y. Niidome, T. Mori, Y. Katayama and T. Niidome, Bioconjugate Chem., 2010, 21, 2049-2054.

26 M. Ajmal, Z. H. Farooqi and M. Siddiq, Korean J. Chem. Eng., 2013, 30, 2030-2036.

27 R. Begum, K. Naseem, E. Ahmed, A. Sharif and Z. H. Farooqi, Colloids Surf., A, 2016, 511, 17-26.

28 R. Begum, Z. H. Farooqi and S. R. Khan, Int. J. Polym. Mater. Polym. Biomater., 2016, 65, 841-852.

29 Y. B. Hamzah, S. Hashim and W. A. W. A. Rahman, J. Polym. Res., 2017, 24, 134.

30 B. R. Saunders, N. Laajam, E. Daly, S. Teow, X. Hu and R. Stepto, Adv. Colloid Interface Sci., 2009, 147, 251-262.

31 K. Naseem, R. Begum, W. Wu, A. Irfan and Z. H. Farooqi, Polym. Rev., 2018, 58, 288-325.

32 K. Naseem, M. A. Ur Rehman and R. Huma, Int. J. Polym. Mater. Polym. Biomater., 2018, 67, 322-332.

33 N. A. Cortez-Lemus and A. Licea-Claverie, Prog. Polym. Sci., 2016, 53, 1-51.

34 F. A. Plamper and W. Richtering, Acc. Chem. Res., 2017, 50, 131-140. 
35 Z. Wang and R. Pelton, Langmuir, 2014, 30, 6763-6767.

36 M. Horecha, V. Senkovskyy, A. Synytska, M. Stamm, A. I. Chervanyov and A. Kiriy, Soft Matter, 2010, 6, 59805992.

37 J. Zhang, H. Chen, L. Xu and Y. Gu, J. Controlled Release, 2008, 131, 34-40.

38 Z. Shen, W. Wei, Y. Zhao, G. Ma, T. Dobashi, Y. Maki, Z. Su and J. Wan, Eur. J. Pharm. Sci., 2008, 35, 271-282.

39 Z. Chen, L. Xu, Y. Liang and M. Zhao, Adv. Mater., 2010, 22, 1488-1492.

40 B. Yıldız, B. Işık and M. Kış, Eur. Polym. J., 2002, 38, 13431347.

41 Y. Pei, J. Chen, L. M. Yang, L. L. Shi and H. J. Luo, J. Shanghai Univ., 2005, 9, 466-470.

42 J. Chen, Y. Pei, L. M. Yang, L. L. Shi and H. J. Luo, Macromol. Symp., 2005, 225, 103-112.

43 G. Fundueanu, M. Constantin and P. Ascenzi, Int. J. Pharm., 2009, 379, 9-17.

44 Z. Cheng, R. Chai, P. Ma, Y. Dai, X. Kang, H. Lian, Z. Hou, C. Li and J. Lin, Langmuir, 2013, 29, 9573-9580.

45 G. Fundueanu, M. Constantin and P. Ascenzi, Acta Biomater., 2009, 5, 363-373.

46 N. S. Satarkar, D. Johnson, B. Marrs, R. Andrews, C. Poh, B. Gharaibeh, K. Saito, K. W. Anderson and J. Z. Hilt, J. Appl. Polym. Sci., 2010, 117, 1813-1819.

47 J. Chen, M. Liu, Q. Huang, L. Huang, H. Huang, F. Deng, Y. Wen, J. Tian, X. Zhang and Y. Wei, Chem. Eng. J., 2018, 337, 82-90.

48 J. Chen, M. Liu, H. Huang, F. Deng, L. Mao, Y. Wen, L. Huang, J. Tian, X. Zhang and Y. Wei, J. Mol. Liq., 2018, 259, 179-185.

49 A. Khan, T. H. Khan, A. M. El-Toni, A. Aldalbahi, J. Alam and T. Ahamad, Mater. Lett., 2019, 235, 197-201.

50 R. Begum, Z. H. Farooqi, K. Naseem, F. Ali, M. Batool, J. Xiao and A. Irfan, Crit. Rev. Anal. Chem., 2018, 1-14.

51 N. H. Cao-Luu, Q. T. Pham, Z. H. Yao, F. M. Wang and C. S. Chern, J. Colloid Interface Sci., 2019, 536, 536-547.

52 H. M. Crowther, B. R. Saunders, S. J. Mears, T. Cosgrove, B. Vincent, S. M. King and G.-E. Yu, Colloids Surf., A, 1999, 152, 327-333.

53 B. R. Saunders and B. Vincent, J. Chem. Soc., Faraday Trans., 1996, 92, 3385-3389.

54 S. Mears, Y. Deng, T. Cosgrove and R. Pelton, Langmuir, 1997, 13, 1901-1906.

55 H. Crowther and B. Vincent, Colloid Polym. Sci., 1998, 276, 46-51.

56 E. Daly and B. R. Saunders, Langmuir, 2000, 16, 5546-5552.

57 Y. Yin, S. Jiao, C. Lang and J. Liu, Soft Matter, 2014, 10, 3374-3385.

58 L. A. Shah, M. Sayed, M. Fayaz, I. Bibi, M. Nawaz and M. Siddiq, Nanotechnol. Environ. Eng., 2017, 2, 14-21.

59 W. Wu, T. Zhou, M. Aiello and S. Zhou, Chem. Mater., 2009, 21, 4905-4913.

60 J. Fang, C. Wang, M. Cao, M. Cheng, J. Shi and Y. Jin, Mater. Lett., 2013, 96, 89-92.
61 B. Sanz, C. Von Bilderling, J. S. Tuninetti, L. Pietrasanta, C. Mijangos, G. S. Longo, O. Azzaroni and J. M. Giussi, Soft Matter, 2017, 13, 2453-2464.

62 Q. Wang, Y. Zhao, Y. Yang, H. Xu and X. Yang, Colloid Polym. Sci., 2007, 285, 515-521.

63 G. Huang, J. Gao, Z. Hu, J. V. S. John, B. C. Ponder and D. Moro, J. Controlled Release, 2004, 94, 303-311.

64 B. Wedel, T. Brändel, J. Bookhold and T. Hellweg, ACS Omega, 2017, 2, 84-90.

65 Z. H. Farooqi and M. Siddiq, J. Dispersion Sci. Technol., 2015, 36, 423-429.

66 W. Wu, S. Chen, Y. Hu and S. Zhou, J. Diabetes Sci. Technol., 2012, 6, 892-901.

67 X. Q. Zhao, T. X. Wang, W. Liu, C. D. Wang, D. Wang, T. Shang, L. H. Shen and L. Ren, J. Mater. Chem., 2011, 21, 7240-7247.

68 L. Hu, S. Gao, X. Ding, D. Wang, J. Jiang, J. Jin and L. Jiang, ACS Nano, 2015, 9, 4835-4842.

69 L. Hu, A. K. Sarker, M. R. Islam, X. Li, Z. Lu and M. J. Serpe, J. Polym. Sci., Part A: Polym. Chem., 2013, 51, 3004-3020.

70 C. D. Sorrell, M. C. Carter and M. J. Serpe, ACS Appl. Mater. Interfaces, 2011, 3, 1140-1147.

71 L. Hu and M. J. Serpe, Polymers, 2012, 4, 134-149.

72 T. Shiroya, N. Tamura, M. Yasui, K. Fujimoto and H. Kawaguchi, Colloids Surf., B, 1995, 4, 267-274.

73 Q. Wu, H. Cheng, A. Chang, W. Xu, F. Lu and W. Wu, Chem. Commun., 2015, 51, 16068-16071.

74 S. Samai, Z. Qian, J. Ling, K. N. Guye and D. S. Ginger, ACS Appl. Mater. Interfaces, 2018, 10, 8976-8984.

75 W. Wu, T. Zhou, J. Shen and S. Zhou, Chem. Commun., 2009, 4390-4392.

76 H. Wang, J. Yi, D. Velado, Y. Yu and S. Zhou, ACS Appl. Mater. Interfaces, 2015, 7, 15735-15745.

77 Q. Wan, Q. Huang, M. Liu, D. Xu, H. Huang, X. Zhang and Y. Wei, Appl. Mater. Today, 2017, 9, 145-160.

78 X. Zhang, S. Wang, L. Xu, L. Feng, Y. Ji, L. Tao, S. Li and Y. Wei, Nanoscale, 2012, 4, 5581-5584.

79 M. Liu, G. Zeng, K. Wang, Q. Wan, L. Tao, X. Zhang and Y. Wei, Nanoscale, 2016, 8, 16819-16840.

80 Y. Shi, M. Liu, F. Deng, G. Zeng, Q. Wan, X. Zhang and Y. Wei, J. Mater. Chem. B, 2017, 5, 194-206.

81 L. Huang, M. Liu, H. Huang, Y. Wen, X. Zhang and Y. Wei, Biomacromolecules, 2018, 19, 1858-1868.

82 M. Fan, F. Wang and C. Wang, Macromol. Biosci., 2018, 18, 1800077.

83 G. Agrawal and R. Agrawal, Small, 2018, 14, 1801724.

84 W. Li, L. Zhang, X. Ge, B. Xu, W. Zhang, L. Qu, C.-H. Choi, J. Xu, A. Zhang and H. Lee, Chem. Soc. Rev., 2018, 47, 56465683.

85 S. S. Suner, M. Sahiner, S. B. Sengel, D. J. Rees, W. F. Reed and N. Sahiner, in Stimuli Responsive Polymeric Nanocarriers for Drug Delivery Applications, Elsevier, 2018, vol. 1, pp. 453500 .

86 M. Wei, Y. Gao, X. Li and M. J. Serpe, Polym. Chem., 2017, 8, 127-143.

87 W. Wu, N. Mitra, E. C. Yan and S. Zhou, ACS Nano, 2010, 4, 4831-4839. 
88 W. Wu, J. Shen, P. Banerjee and S. Zhou, Biomaterials, 2010, 31, 7555-7566.

89 Z. Zhang, J. Wang, X. Nie, T. Wen, Y. Ji, X. Wu, Y. Zhao and C. Chen, J. Am. Chem. Soc., 2014, 136, 7317-7326.

90 L. Yu, A. Dong, R. Guo, M. Yang, L. Deng and J. Zhang, ACS Biomater. Sci. Eng., 2018, 4, 2424-2434.

91 J. Cao, H. Niu, J. Du, L. Yang, M. Wei, X. Liu, Q. Liu and J. Yang, J. Mater. Sci.: Mater. Med., 2018, 29, 169.

92 B. Sung, S. Shaffer, M. Sittek, T. Alboslemy, C. Kim and M. H. Kim, J. Visualized Exp., 2016, 1, 53680-53685.

93 H. Wang, J. Yi, S. Mukherjee, P. Banerjee and S. Zhou, Nanoscale, 2014, 6, 13001-13011.

94 F. Zhao, X. Qin and S. Feng, RSC Adv., 2016, 6, 100511100518.

95 L. Pan, G. Zhai, X. Yang, H. Yu and C. Cheng, ACS Omega, 2019, 4, 3933-3945.

96 G. Sharma, A. Kumar, M. Naushad, A. García-Peñas, H. Ala'a, A. A. Ghfar, V. Sharma, T. Ahamad and F. J. Stadler, Carbohydr. Polym., 2018, 202, 444-453.

97 C. B. Godiya, X. Cheng, D. Li, Z. Chen and X. Lu, J. Hazard. Mater., 2019, 364, 28-38.

98 Y. Liu, H. Huang, D. Gan, L. Guo, M. Liu, J. Chen, F. Deng, N. Zhou, X. Zhang and Y. Wei, Ceram. Int., 2018, 44, 1857118577.
99 X. Zhang, Q. Huang, M. Liu, J. Tian, G. Zeng, Z. Li, K. Wang, Q. Zhang, Q. Wan and F. Deng, Appl. Surf. Sci., 2015, 343, 19-27.

100 X. Zhang, Q. Huang, F. Deng, H. Huang, Q. Wan, M. Liu and Y. Wei, Appl. Mater. Today, 2017, 7, 222-238.

101 Q. Huang, M. Liu, J. Chen, Q. Wan, J. Tian, L. Huang, R. Jiang, Y. Wen, X. Zhang and Y. Wei, Appl. Surf. Sci., 2017, 419, 35-44.

102 Q. Huang, M. Liu, L. Mao, D. Xu, G. Zeng, H. Huang, R. Jiang, F. Deng, X. Zhang and Y. Wei, J. Colloid Interface Sci., 2017, 499, 170-179.

103 K. Naseem, R. Begum, W. Wu, M. Usman, A. Irfan, A. G. AlSehemi and Z. H. Farooqi, J. Mol. Liq., 2019, 277, 522-531.

104 K. Naseem, Z. H. Farooqi, R. Begum, M. Ghufran, M. Z. U. Rehman, J. Najeeb, A. Irfan and A. G. Al-Sehemi, J. Mol. Liq., 2018, 268, 229-238.

105 G. Zeng, L. Huang, Q. Huang, M. Liu, D. Xu, H. Huang, Z. Yang, F. Deng, X. Zhang and Y. Wei, Appl. Surf. Sci., 2018, 459, 588-595.

106 L. Nyström, A. A. Strömstedt, A. Schmidtchen and M. Malmsten, Biomacromolecules, 2018, 19, 3456-3466.

107 M. Qasim, N. Udomluck, J. Chang, H. Park and K. Kim, Int. J. Nanomed., 2018, 13, 235. 